\title{
Large-eddy simulation of neutral atmospheric boundary layer flow over heterogeneous surfaces: Blending height and effective surface roughness
}

\author{
Elie Bou-Zeid \\ Department of Geography and Environmental Engineering and Center for Environmental and Applied Fluid Mechanics, \\ Johns Hopkins University, Baltimore, Maryland, USA

\section{Charles Meneveau} \\ Department of Mechanical Engineering and Center for Environmental and Applied Fluid Mechanics, Johns Hopkins \\ University, Baltimore, Maryland, USA \\ Marc B. Parlange \\ Department of Geography and Environmental Engineering and Center for Environmental and Applied Fluid Mechanics, \\ Johns Hopkins University, Baltimore, Maryland, USA
}

Received 10 July 2003; revised 9 November 2003; accepted 3 December 2003; published 17 February 2004.

[1] A new generation large-eddy simulation (LES), based on a Lagrangian scaledependent dynamic subgrid model, is applied to neutral atmospheric flow over heterogeneous land surfaces. This LES is faithful to the physics of the interaction of the lower atmosphere and the land surface based on classical validation tests of the simulated mean wind profile and the atmospheric turbulence. Simulations of the atmospheric boundary layer $(\mathrm{ABL})$ over heterogeneous land surfaces with a range of characteristic lengths and surface roughness values are performed, each simulated surface consisting of equal-size stripes of different roughness. The simulated mean wind profiles are analyzed to identify the height of the blending layer and used to develop a relationship between blending layer height and characteristic surface length scales. For hydrologic and atmospheric applications where the regional-scale surface roughness needs to be known, the analysis is extended to derive an effective surface roughness knowing local surface patch roughness values. INDEX TERMS: 3322 Meteorology and Atmospheric Dynamics: Land/ atmosphere interactions; 1818 Hydrology: Evapotranspiration; 3337 Meteorology and Atmospheric Dynamics: Numerical modeling and data assimilation; 3307 Meteorology and Atmospheric Dynamics: Boundary layer processes; KEYWORDS: atmospheric boundary layer, blending height, effective surface roughness, large-eddy simulation, mesoscale model, subgrid scale

Citation: Bou-Zeid, E., C. Meneveau, and M. B. Parlange (2004), Large-eddy simulation of neutral atmospheric boundary layer flow over heterogeneous surfaces: Blending height and effective surface roughness, Water Resour. Res., 40, W02505, doi:10.1029/2003WR002475.

\section{Introduction}

[2] The determination of regional-scale surface fluxes of momentum, sensible heat and water vapor over complex terrain remains a basic challenge in hydrology and the atmospheric sciences. Monin-Obukhov similarity theory (MOS) [Monin and Obukhov, 1954] is the main practical tool for the computation of surface fluxes using measurements in the atmospheric surface layer [e.g., Parlange et al., 1995]. MOS was initially developed for homogeneous surfaces, but it has been commonly used for heterogeneous surfaces as well. The applicability of this similarity theory over heterogeneous surfaces can be attributed to the strong mixing effects of atmospheric turbulence [Brutsaert, 1998]. Therefore MOS can be used for heterogeneous surfaces only above a height at which surface and local disturbances

Copyright 2004 by the American Geophysical Union. 0043-1397/04/2003WR002475\$09.00 have been blended out; this height is known as the blending height $h_{\mathrm{b}}$ [Wieringa, 1976]. Another main characteristic of $\mathrm{ABL}$ flows that allows the use of MOS for nonhomogeneous surfaces is the small extent of vertical length scales vis-à-vis horizontal length scales [Brutsaert, 1998]. In this regard, it is important to distinguish between heterogeneities in terms of scale. The largest eddies in the ABL are of the same scale as the depth of the ABL; therefore for heterogeneities smaller than the ABL depth, turbulent mixing is important and has a strong blending effect. On the other hand, for heterogeneities larger than the depth of the ABL, mixing and patch interaction is mainly due to mean circulation. Field experiments found the similarity theory to be accurate in obtaining the regional-scale evaporation, momentum flux, and sensible heat flux over heterogeneous surfaces that appear statistically homogeneous at a regionalscale [Brutsaert et al., 1989; Parlange and Brutsaert, 1989, 1993]. However, in experiments conducted at the HAPEXMOBILHY site in southeastern France over areas with 
large, distinct, and abrupt changes in land use, it was found that the similarity theory did not always work [Brutsaert and Parlange, 1992]. Despite the wide use of MOS, several questions related to flow over heterogeneous terrain are yet to be addressed: (1) How are internal boundary layers formed over patches blended and what is the mixing height above which turbulence blends out the effects of surface heterogeneity? (2)What is the effect of surface heterogeneity on surface shear stress and consequently on land atmosphere interactions? (3) Can these effects be parameterized succinctly and accurately?

[3] The need to answer these questions becomes obvious when mesoscale and global models are considered. In these models, significant variations in surface conditions occur at scales smaller than the grid scale [Avissar and Pielke, 1989; Koster and Suarez, 1992; Intergovernmental Panel on Climate Change, 1997]. Hence a proper parameterization of heterogeneity that would yield good estimates of average transport is of critical importance. Several approaches have been attempted. Methods that simply use the average of surface roughness, temperature or humidity, might not capture the effect of abrupt changes in surface conditions. Other approaches use higher order statistics such as frequency distributions [Avissar, 1991, 1992]; these approaches do not account for heterogeneity scales and interactions between patches. For example, it will be shown in this work that simulations with the same probability density function for surface roughness can yield very different land-atmosphere interaction dynamics (as depicted by the effective surface roughness for example), due to variations in the heterogeneity scale and the resulting variations in patch interactions.

[4] In addition to experimental studies, Reynolds-Averaged Navier-Stokes (RANS) simulations have been used to understand land-atmosphere interaction and to formulate a method to account for subgrid-scale heterogeneities [Mason, 1988; Claussen, 1991; Wood and Mason, 1991; Schmid and Bünzly, 1995; Goode and Belcher, 1999]. RANS solves the ensemble averaged Navier-Stokes equations; therefore a closure model is needed to parameterize the turbulent flux term. Since turbulence is parameterized rather than simulated in RANS, this technique can only give information about the average quantities and hence cannot dynamically capture nonlocal transport over abrupt changes in surface conditions.

[5] More recently, large-eddy simulation has become increasingly popular as a tool for a physical understanding of the dynamics of the blending phenomena over heterogeneous surfaces [Hechtel et al., 1990; Shen and Leclerc, 1995; Khanna and Brasseur, 1997; Avissar et al., 1998; Albertson and Parlange, 1999a, 1999b; Glendening and Lin, 2002]. This paper continues this previous body of work by using LES, with an up-to-date model for subgrid-scale stresses, to test a new parameterization for heterogeneous surfaces.

\section{Large-Eddy Simulation}

[6] In large-eddy simulation, the basic premise is that the largest eddies contain most of the energy and are responsible for most of the transport of momentum and scalars. Hence the LES technique consists of solving the NavierStokes equations with eddies smaller than the filter size left out. Eddies larger than the filter size can be resolved. The solution of the filtered Navier-Stokes equations yields the filtered (resolved) velocity vector $\tilde{\mathbf{u}}(\mathbf{x}, \mathrm{t})$, which represents a spatial average of the actual velocity vector $\mathbf{u}(\mathbf{x}, t)$. Note that the tilde will be used throughout this paper to indicate the filtering operation (at the grid scale $\Delta$ ) or to refer to a filtered variable, a caret will denote filtering at double the grid scale $(2 \Delta)$, a prime will denote the subgrid-scale (SGS) components of the variables. Statistical averaging will be denoted by angle brackets. Brackets followed by dimensions subscripts will denote averaging in all the indicated dimensions; for example, $\langle\mathbf{u}\rangle_{\mathrm{x}, \mathrm{y}}$ is the velocity averaged over the $\mathrm{x}$ and $\mathrm{y}$ directions.

[7] The spatial filtering of the Navier-Stokes equations gives rise to a subgrid-scale (SGS) flux term $\sigma_{\mathrm{ij}}=$ $\widetilde{u_{i} u_{j}}-\widetilde{u_{i}} \widetilde{u}_{j}$; it represents the effect of small eddies that are filtered out on the resolved scales of motion. An important effect of the small eddies is to pass the cascading energy to smaller and smaller scales until that energy can be dissipated by molecular viscosity, a phenomenon not explicitly resolved in LES.

[8] In this work the viscous term can be neglected due to the extremely high Reynolds number of atmospheric flows and the modeling of the wall layer (as opposed to resolving the viscous sublayer). The Coriolis term can also be dropped since the paper mainly looks at the physics in a layer that does not exceed $300 \mathrm{~m}$ in height. The filtered Navier-Stokes equation in rotational form [Orszag and Pao, 1974] and the continuity equation can then be written as

$$
\frac{\partial \tilde{u}_{i}}{\partial t}+\tilde{u}_{j}\left(\frac{\partial \tilde{u}_{i}}{\partial x_{j}}-\frac{\partial \tilde{u}_{j}}{\partial x_{i}}\right)=-\frac{1}{\rho} \frac{\partial \tilde{p}^{*}}{\partial x_{i}}-\frac{\partial}{\partial x_{j}} \tau_{i j}+\widetilde{F}_{i}, \frac{\partial \tilde{u}_{i}}{\partial x_{i}}=0
$$

The rotational form is used for kinetic energy and mass conservation [Orszag and Pao, 1974]. $F_{i}$ is the external forcing; in this study for example, it is the mean streamwise pressure forcing. $\tau_{i j}$ is the deviatoric part of the SGS stress tensor defined as

$$
\tau_{i j}=\sigma_{i j}-\frac{1}{3} \sigma_{k k} \delta_{i j}
$$

[9] The pressure term $\widetilde{p}^{*}=\tilde{p}+\frac{1}{3} \rho \sigma_{k k}+\frac{1}{2} \rho \widetilde{u}_{j} \widetilde{u}_{j}$ represents a dynamic variable that is formulated so that the velocity field remains divergence free. Taking the divergence of the above equation and using continuity results in a Poisson equation for pressure that can be solved for $\widetilde{p}^{*}$.

[10] The code (described in detail by Albertson and Parlange [1999b] and Porté-Agel et al. [2000]) uses a pseudo-spectral approach in the horizontal directions and a second order accurate centered-differences scheme in the vertical direction. The centered-differences scheme necessitates the use of a staggered grid in the vertical direction; this entails storing the variables either at heights $n d z$ or $(n-$ $1 / 2) d z$, where $n$ goes from 0 to $N z$ ( $N z$ is the number of vertical grid points and $d z$ is the vertical mesh spacing). The fully explicit second order accurate Adams-Bashforth scheme is used for time advancement. Full dealiasing of the convective terms is achieved by padding and truncation using the $3 / 2$ rule [Orszag, 1970]. This is needed in the present study since aliasing errors, which affect the smallest resolved scales used to compute the dynamic Smagorinsky 
coefficient (see below), might be significantly detrimental to the accuracy of SGS parameterization.

\section{SGS Modeling}

[11] The usefulness of LES in atmospheric applications has significantly increased in the past decade due to the increase in computing power and the improvement in SGS parameterizations. As previously discussed, solving the LES equations requires expressing the deviatoric SGS stress, $\tau_{i j}$, in terms of the resolved velocity. The SGS parameterization most often used in traditional LES of ABL flows is the Smagorinsky model [Smagorinsky, 1963]; it uses viscous analogy and the mixing length approach and expresses the SGS flux as a function of the resolved strain rate tensor $\tilde{S}_{i j}=0.5\left(\partial_{j} \widetilde{u}_{i}+\partial_{i} \widetilde{u}_{j}\right)$ using

$$
\tau_{i j}^{s m a g}=-2 \nu_{T} \tilde{S}_{i j}=-2\left(c_{s, \Delta} \Delta\right)^{2}\left|\tilde{S}_{i j}\right| \tilde{S}_{i j},
$$

where $\Delta$ is the grid width and $\nu_{T}$ is the eddy viscosity. The only unknown in the above expression is the Smagorinsky coefficient $c_{s, \Delta}$. The traditional approach is to use a constant coefficient estimated from the theory of isotropic homogeneous turbulence to be about 0.17 [Lilly, 1967]. However, and most importantly for ABL applications, the Smagorinsky model fails when the grid-scale approaches the limits of the inertial range, such as in the vicinity of solid boundaries. It is precisely in such areas that subgrid-scale fluxes contribute a significant share of the total fluxes and where an accurate model is needed. The Smagorinsky coefficient must decrease close to the surface, and attempts to impose empirical wall damping functions have shown that the model is over dissipative regardless of the damping function used [Mason and Thomson, 1992]. In summary, for faithful modeling of SGS processes, $c_{s, \Delta}$ cannot be assumed to be flow and situation independent unless $\Delta$ is contained well inside an ideal inertial range of locally isotropic and homogeneous turbulence.

\subsection{Dynamic Model}

[12] A major improvement in LES technique has been the introduction of the dynamic SGS model [Germano et al., 1991]. The dynamic approach consists of using the resolved scales to "measure" the model coefficient during the simulation. As reviewed by Meneveau and Katz [2000], the approach uses the assumption of scale invariance, $c_{s, 2 \Delta}=$ $c_{s, \Delta}$, by applying the coefficient measured from the resolved scales to the subgrid-scale range. However, scale invariance near the grid-filter scale $\Delta$ does not always hold. The coefficient depends strongly on scale when $\Delta$ tends to the integral scale (as occurs in the first few grid points near the ground in LES of ABL flows). To account for scale effects, scale-dependent formulations have been successfully implemented for ABL flows [Porté-Agel et al., 2000]. A power law behavior is assumed for the scale dependence of $c_{s}: c_{s, \Delta} \sim \Delta^{\Phi}$ or $c_{s, 2 \Delta} \sim \beta \Delta^{\Phi}=2^{\Phi} \Delta^{\Phi}, \Phi=0$ (i.e., $\beta=1$ ) corresponding to the scale-invariant standard dynamic model.

[13] In the work of Porté-Agel et al. [2000], the coefficient $\beta$ itself was measured dynamically through an additional filtering operation at a scale $4 \Delta$. That paper also showed that the use of the scale-dependent dynamic model for simple LES of neutrally stable ABL gives substantial

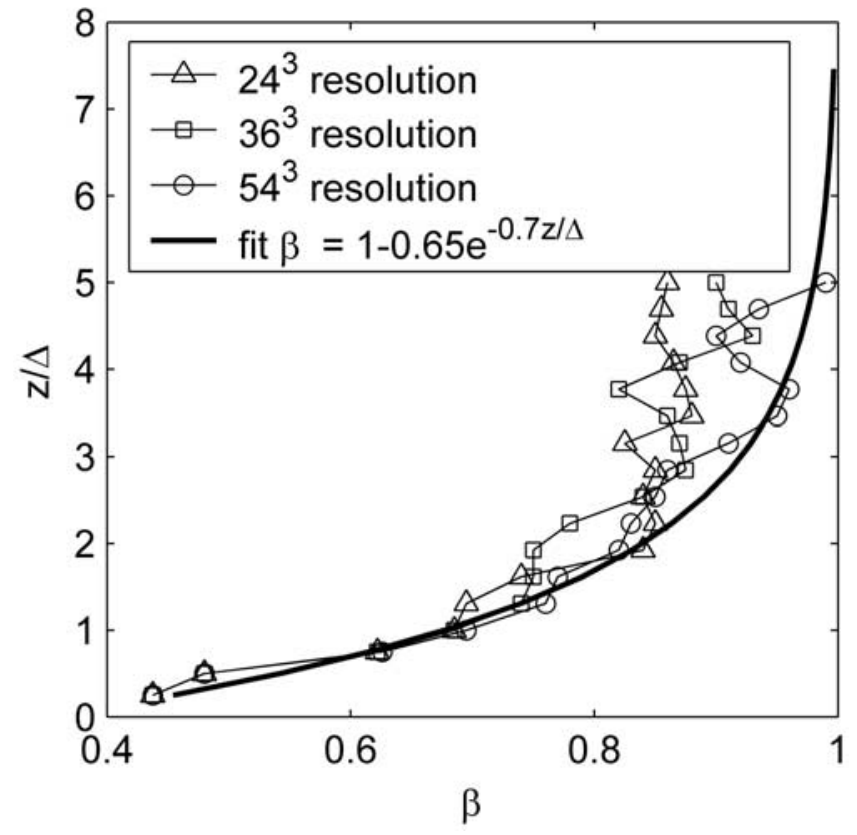

Figure 1. Scaling factor $\beta=2^{\Phi}$ versus $\mathrm{z} / \Delta$ from PortéAgel et al. [2000] and fit used in this work.

improvements over both the traditional Smagorinsky model with wall-damping functions and the scale-invariant dynamic model.

[14] In this work, for computational simplicity, a dynamic computation of $\beta$ is not performed. Instead, the vertical profile of $\beta$ as a function of $\mathrm{z} / \Delta$ is taken from Porté-Agel et al. [2000]. Figure 1 depicts the smooth fit describing the already computed dependence of $\beta$ upon $z / \Delta$ for the case of neutral ABL over rough terrain. Note that this function for $\beta$ was verified only for neutral atmospheres and uniform surface roughness. The relation was tested in this work for a wide range of surface roughness values and it yielded realistic velocity profiles in the log region which suggests that it holds well for the surfaces simulated in this paper. By using the function for the case with patches, the possible effects of transitions among patches on $\beta$ is being neglected. It is reasonable to expect these effects to be small since the relation only has to hold between the filter size $\Delta$ and the second test filter size $4 \Delta$.

\subsection{Lagrangian Scale-Dependent SGS Model}

[15] The dynamic model, even in its scale-dependent formulation, yields a coefficient with strong fluctuations and averaging is needed for numerical stability. The traditional approach consists of averaging over homogeneous directions, for example horizontal planes in ABL flows. This requirement for homogeneous directions in the flow field and the concomitant inability to handle complex geometries renders the use of this model questionable in studying the effect of surface heterogeneity. Instead, a new version of the so-called Lagrangian dynamic SGS model [Meneveau et al., 1996] is implemented.

[16] The approach is based on the dynamic model but averages are obtained in time along fluid path lines rather than over homogeneous directions. The Lagrangian model is very well suited for applications with heterogeneous spatial conditions since it preserves local variability, pre- 
serves Galilean invariance, and does not require homogeneous directions. The model starts from the Germano identity [Germano et al., 1991] that relates SGS stresses at different scales

$$
L_{i j}=T_{i j}-\hat{\sigma}_{i j}=\widehat{\tilde{u}}_{i} \tilde{u}_{j}-\widehat{\tilde{u}}_{i} \widehat{\tilde{u}}_{j},
$$

where $T_{i j}$ is the SGS stress at a scale $2 \Delta$ and $L_{i j}$ is the resolved SGS stress tensor defined from scales intermediate between $\Delta$ and $2 \Delta . L_{i j}$ can be computed exactly from the resolved velocity field. Using the Smagorinsky model to express the deviatoric parts of the SGS stresses at the scales $\Delta$ and $2 \Delta$, and assuming that the $c_{\mathrm{s}}$ coefficient does not fluctuate strongly in space to justify extracting it from the test-filtering operation [Ghosal et al., 1995; Meneveau et al., 1996] results in the following expressions:

$$
\widehat{\sigma_{i j}^{D}}=-2 c_{s, \Delta}^{2} \Delta^{2}|\widehat{\widetilde{S}}| \widetilde{S}_{i j}, \quad T_{i j}^{D}=-2 c_{s, 2 \Delta}^{2}(2 \Delta)^{2}|\widehat{\widetilde{S}}| \widehat{\widetilde{S}}_{i j}
$$

The superscript $\mathrm{D}$ denotes the trace free part of the tensor, for example $T_{i j}{ }^{D}=T_{i j}-1 / 3\left(T_{k k} \delta_{i j}\right)$. Replacing in equation (4) yields an error induced by the use of the Smagorinsky model. This error is

$$
\mathrm{e}_{\mathrm{ij}}=L_{i j}^{D}-\left(T_{i j}^{D}-\widehat{\tau}_{i j}\right)=L_{i j}^{D}-c_{s, \Delta}^{2} M_{i j}
$$

where $M_{i j}=2 \Delta^{2}\left[|\widehat{\widetilde{S}}| \widetilde{S}_{i j}-4 \beta|\widehat{\widetilde{S}}| \widehat{\widetilde{S}}_{i j}\right]$ and $\beta=c_{s, 2 \Delta}^{2} / c_{s, \Delta}^{2}$ as already defined. In the Lagrangian SGS model, the coefficient $c_{S, \Delta}$ is obtained by minimizing the weighted time average of the squared error over path lines

$$
\mathrm{E}=\int_{-\infty}^{t} \mathrm{e}_{i j}\left(\mathbf{z}\left(t^{\prime}\right), t^{\prime}\right) \mathrm{e}_{i j}\left(\mathbf{z}\left(t^{\prime}\right), t^{\prime}\right) \mathrm{W}\left(\mathrm{t}-\mathrm{t}^{\prime}\right) \mathrm{dt}^{\prime},
$$

where $\mathbf{z}\left(\mathrm{t}^{\prime}\right)$ are the previous positions of the fluid elements. Setting the partial derivative of $\mathrm{E}$ with respect to $c_{\mathrm{s}, \Delta}^{2}$ to zero results in an expression for $c_{\mathrm{s}, \Delta}^{2}$ (note that the resulting contraction of $L_{i j}$ with $M_{i j}$ eliminates the need to distinguish between $L_{i j}$ and $L_{i j}^{\mathrm{D}}$, since $M_{i j}$ is a deviatoric (traceless) tensor)

$$
c_{s, \Delta}^{2}=\frac{I_{L M}}{I_{M M}}
$$

where $I_{L M}=\int_{-\infty}^{t} L_{i j} M_{i j}\left(\mathbf{z}\left(t^{\prime}\right), t\right) W\left(t-t^{\prime}\right) d t^{\prime}$ and $I_{M M}=$ $\int_{-\infty}^{t} M_{i j} M_{i j}\left(\mathbf{z}\left(t^{\prime}\right), t^{\prime}\right) W\left(t-t^{\prime}\right) d t^{\prime} . W(t)$ is a relaxation function that allows the model to allocate a higher weight to its recent history. A choice of an exponential form, $W\left(t-t^{\prime}\right)=$

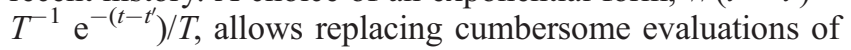
backward time integrals with forward relaxation-transport equations. On the basis of DNS results and dimensional self-consistency [Meneveau et al., 1996], the timescale $\mathrm{T}$ is chosen as $T=1.5 \Delta\left(\mathrm{I}_{L M} \mathrm{I}_{M M}\right)^{-1 / 8}$. The relaxation transport equations thus obtained for $\mathrm{I}_{L M}$ and $\mathrm{I}_{M M}$ are:

$$
\begin{gathered}
\frac{D I_{L M}}{D t}=\frac{\partial I_{L M}}{\partial t}+\widetilde{\boldsymbol{u}} \cdot \nabla I_{L M}=\frac{1}{T}\left(L_{i j} M_{i j}-I_{L M}\right), \\
\frac{D I_{M M}}{D t}=\frac{\partial I_{M M}}{\partial t}+\widetilde{\boldsymbol{u}} \cdot \nabla I_{M M}=\frac{1}{T}\left(M_{i j} M_{i j}-I_{M M}\right) .
\end{gathered}
$$

[17] These equations can be readily included in the numerical LES model using low-order numerical approximations and the resultant formulation is particularly simple and efficient:

$$
\begin{gathered}
I_{M M}^{n+1}(\boldsymbol{x})=\varepsilon\left[M_{i j} M_{i j}\right]^{n+1}(\boldsymbol{x})+(1-\varepsilon) I_{M M}^{n}\left(\boldsymbol{x}-\widetilde{\boldsymbol{u}}^{n} \Delta t\right), \\
I_{L M}^{n+1}(\boldsymbol{x})=\mathrm{H}\left\{\varepsilon\left[L_{i j} M_{i j}\right]^{n+1}(\boldsymbol{x})+(1-\varepsilon) I_{L M}^{n}\left(\boldsymbol{x}-\widetilde{\boldsymbol{u}}^{n} \Delta t\right)\right\}, \\
\text { where } \varepsilon=\frac{\Delta t / T^{n}}{1+\Delta t / T^{n}}, \quad T^{n}=1.5 \Delta\left(I_{L M}^{n} I_{M M}^{n}\right)^{-1 / 8}, \\
\text { and } \quad \mathrm{H}\{\mathrm{x}\}=\text { ramp function }=\left|\begin{array}{ll}
x & \text { if } \quad x \geq 0 \\
0 & \text { otherwise. }
\end{array}\right|
\end{gathered}
$$

$\Delta t$ is the time step. The equations are advanced with periodic boundary conditions for $I_{L M}$ and $I_{M M}$ in the horizontal directions. At the lower and upper boundaries, zero-gradient (homogeneous Neumann) boundary conditions are imposed, i.e., the values at the boundary are set equal to the values at the closest node inside the domain. The model coefficient does not need to be updated at every time step of the LES code. In this work, $c_{s}$ is computed every fifth time step and tests showed that no significant improvements can be obtained by updating $c_{s}$ more frequently. The computational cost of the scale-dependent dynamic Lagrangian SGS model implemented here exceeds the cost of the traditional Smagorinsky model (imposed $c_{s}$ ) by only $11 \%$. The authors consider that the improved results obtained justify the additional cost. It is also highly unlikely that a Smagorinsky model with a higher resolution could yield equivalent results since the $11 \%$ increase in the cost is equivalent to a mere $3.5 \%$ increase in resolution $\left(1.11^{1 / 3}\right)$ in each direction, even without considering the increased constraint on the time step associated with increased resolution.

\section{A Suite of Simulations}

[18] To understand the dynamics of land-atmosphere coupling over heterogeneous surfaces and the effects of patch scale on ABL dynamics, a suite of simulations was performed. In all the simulations, the flow characteristics were kept unchanged and only the streamwise length of the patches and their surface roughness were varied. While such uniform stripes are highly idealized representations of real complex heterogeneous terrains, they are good surrogate of heterogeneity characterized by a single length scale. Future extensions of this work will consider more complex patch configurations. Figure 2 depicts the simulation domain and the main parameters used in the simulations. It represents the 4-patch case. Cases with a higher or lower number of patches have a similar configuration with a different number of alternating low roughness and high roughness patches. Table 1 details the simulations characteristics.

[19] The boundary conditions in the horizontal directions are periodic. Hence the flow that exits at the downstream boundary of the domain is fed back at the upstream boundary. This implies that the domain simulated actually consists of an infinite number of alternating patches in the streamwise direction (x). Similarly, the periodic boundary conditions in the cross-stream direction (y) imply that the 


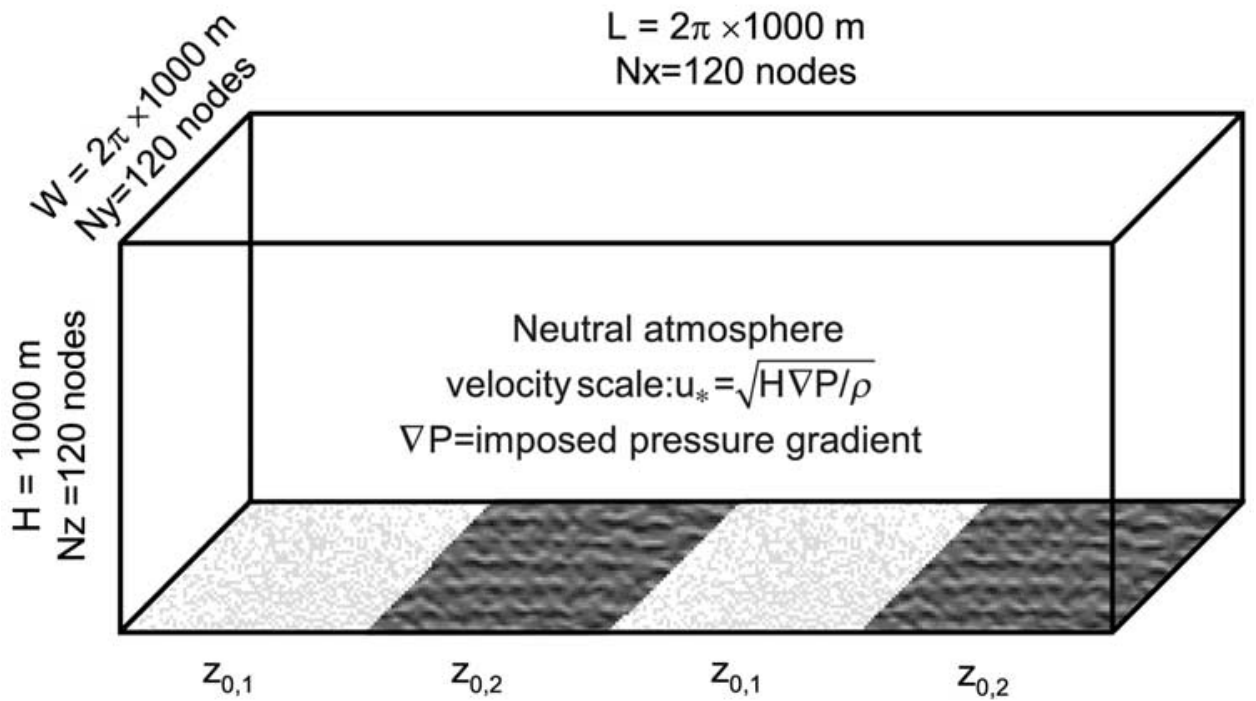

Figure 2. Simulation domain (not to scale).

patches are of infinite width in that direction. The transition between patches is abrupt. A stress free condition is imposed at the top of the domain by setting

$$
\partial_{3} \widetilde{u}_{1,2}=\widetilde{u}_{3}=0
$$

where 1,2 , and 3 refer to the streamwise, cross-stream and vertical directions, respectively. At the bottom of the domain, the vertical velocity is set to 0 at the surface and a stress boundary condition is imposed through the logarithmic law-of-the-wall.

[20] A common approach used in many studies [Moeng, 1984; Mason and Callen, 1986; Schmidt and Schumann, 1989; Albertson and Parlange, 1999b] involving heterogeneous surfaces is to impose the law-of-the-wall in a strictly local sense, i.e.,

$$
\tau_{w}=-\left[\frac{\kappa}{\ln \left(z / z_{o}\right)}\right]^{2}\left(\widetilde{u}_{1}^{2}+\widetilde{u}_{2}^{2}\right),
$$

where $\kappa$ is the von Karman constant (0.4) and $z=d z / 2$ is the height of the first streamwise velocity node where the velocities used in equation (13) are evaluated. Note that $\tau_{w}$ is in fact the kinematic stress $\tau / \rho$ (the squared friction velocity). The use of this relation imposes an average stress obtained from LES

$$
\left\langle\tau_{w}^{L E S}\right\rangle=-\left[\frac{\kappa}{\ln \left(z / z_{o}\right)}\right]^{2}\left(\left\langle\widetilde{u}_{1}^{2}\right\rangle+\left\langle\widetilde{u}_{2}^{2}\right\rangle\right) .
$$

However, the log-law was developed and validated to be used in an average sense, i.e.,

$$
\left\langle\tau_{w}^{\log -\text { law }}\right\rangle=-\left[\frac{\kappa}{\ln \left(z / z_{o}\right)}\right]^{2}\left\langle\widetilde{u}_{1}\right\rangle^{2},
$$

where the mean cross-stream component $\left\langle\tilde{u}_{2}\right\rangle=0$. Since the velocity at $z=d z / 2$ fluctuates, $\left\langle\tilde{u}_{1}^{2}\right\rangle>\left\langle\tilde{u}_{1}\right\rangle^{2}$ (Schwartz inequality); thus $\left.\left\langle\tau_{w}^{L E S}\right\rangle\right\rangle\left\langle\tau_{w}^{l o g}\right.$-law $\rangle$. Therefore imposing the wall stress in a local sense through equation (13) leads to increased average stresses for a given near-wall velocity. In LES with a prescribed pressure gradient and mean stress, this would yield a slower flow near the surface. The alternative, namely to use the average velocity $\left\langle u_{1}\right\rangle$, is not meaningful and sometimes not feasible in simulations with heterogeneous or complex surface conditions.

[21] However, filtering the local velocity (at $z=d z / 2$ ) at a scale $2 \Delta$ significantly reduces the small-scale fluctuations so that the velocity variance becomes quite small. A formulation that does not require averaging can be derived using this filtered local velocity to impose a stress

\begin{tabular}{|c|c|}
\hline Characteristic & Value/Description \\
\hline Scaling height $H$ & $1000 \mathrm{~m}$ \\
\hline Horizontal mesh spacing, $d x$ and $d y$ & $52 \mathrm{~m}$ \\
\hline Vertical mesh spacing, $d z$ & $8.4 \mathrm{~m}$; as previously discussed, some variables are stored at $(n-1 / 2) d z$ \\
\hline Number of grid points & $120^{3} \sim 1.7$ million points \\
\hline Initial conditions & modified logarithmic profile with a randomly imposed TKE \\
\hline Warm up period & warm-up simulations are run until the stress profile adjusts to a straight line reaching 1 at the surface \\
\hline Simulation time step & 0.00012 (in nondimensional time units) about 0.25 seconds if normalized using $u^{*}=0.5 \mathrm{~m} / \mathrm{s}$ \\
\hline Number of simulation time steps & 80,000 \\
\hline Output sampling frequency & every 10 time steps \\
\hline
\end{tabular}

$$
\tau_{w}(x, y)=-\left[\frac{\kappa}{\ln \left(z / z_{o}\right)}\right]^{2}\left(\widehat{\widetilde{u}}_{1}^{2}(x, y, d z / 2)+\widehat{\widetilde{u}}_{2}^{2}(x, y, d z / 2)\right) .
$$

Table 1. Simulation Characteristics 
Table 2. Simulation Cases

\begin{tabular}{lcrcr}
\hline Simulation & $\begin{array}{c}\text { Number of } \\
\text { Patches }\end{array}$ & $L_{\mathrm{p}}, \mathrm{m}$ & $z_{\mathrm{o}, 1}, z_{\mathrm{o}, 2}, \mathrm{~m}$ & $z_{\mathrm{o}, 2} / z_{\mathrm{o}, 1}$ \\
\hline A1 & 1 & infinite & 0.1 & - \\
A2 & 2 & 3140 & $0.025,0.25$ & 10 \\
A4 & 4 & 1570 & $0.025,0.25$ & 10 \\
A6 & 6 & 1045 & $0.025,0.25$ & 10 \\
A8 & 8 & 785 & $0.025,0.25$ & 10 \\
A12 & 12 & 523 & $0.025,0.25$ & 10 \\
A20 & 20 & 314 & $0.025,0.25$ & 10 \\
A30 & 30 & 209 & $0.025,0.25$ & 10 \\
B2 & 2 & 3140 & $0.1,1$ & 10 \\
B4 & 4 & 1570 & $0.1,1$ & 10 \\
B8 & 8 & 785 & $0.1,1$ & 10 \\
B20 & 20 & 314 & $0.1,1$ & 10 \\
C2 & 2 & 3140 & $0.01,1$ & 100 \\
C4 & 4 & 1570 & $0.01,1$ & 100 \\
C8 & 8 & 785 & $0.01,1$ & 100 \\
C20 & 20 & 314 & $0.01,1$ & 100 \\
D2 & 2 & 3140 & $0.001,0.1$ & 100 \\
D4 & 4 & 1570 & $0.001,0.1$ & 100 \\
D8 & 8 & 785 & $0.001,0.1$ & 100 \\
D20 & 20 & 314 & $0.001,0.1$ & 100 \\
\hline
\end{tabular}

Subsequently, the stress is partitioned into its streamwise and cross-stream components in the usual manner:

$$
\tau_{i, 3}^{\text {wall }}(x, y)=\tau_{w}\left[\frac{\widehat{\widetilde{u}}_{i}(x, y, d z / 2)}{\sqrt{\widehat{\widetilde{u}}_{1}^{2}+\widehat{\widetilde{u}}_{2}^{2}}}\right] \quad i=1,2 .
$$

[22] This formulation is applicable in heterogeneous or complex terrain since the filtering preserves large-scale variations. Tests of this formulation indicate that it yields an average stress that is very close to the stress predicted by the average law-of-the-wall formulation for homogeneous surfaces.

\section{Patch Characteristics}

[23] The roughness of the surfaces was varied to examine the effects of average roughness and the effects of abrupt changes in roughness (i.e., magnitude of roughness jumps). The values of $z_{\mathrm{o}}$ were varied from 0.1 to $100 \mathrm{~cm}$. This is an adequate range and represents surfaces varying in roughness from bare lands to forests. In each case, only two roughness values $z_{\mathrm{o}, 1}$ and $z_{\mathrm{o}, 2}$ were used. The roughness ratio $z_{0,2} / z_{0,1}$ was set to 10 or 100 . Four groups of simulations were performed. The first group consisted of eight cases, with one reference case having no patches, i.e., a homogeneous surface, while the other cases used patches of different lengths $L_{\mathrm{p}}$. The other three groups consisted each of 4 cases for different $L_{\mathrm{p}}$ and different combinations of $z_{\mathrm{o}, 1}$ and $z_{\mathrm{o}, 2}$. Table 2 lists the different scenarios and their characteristics.

\section{Code Validation}

[24] The code with the new surface boundary condition and Lagrangian scale-dependent SGS model was validated. Results from the homogeneous simulation (A1) were used since the literature for LES over homogeneous surfaces is more abundant for comparison of simulation performance. Figure 3 shows the nondimensional streamwise velocity

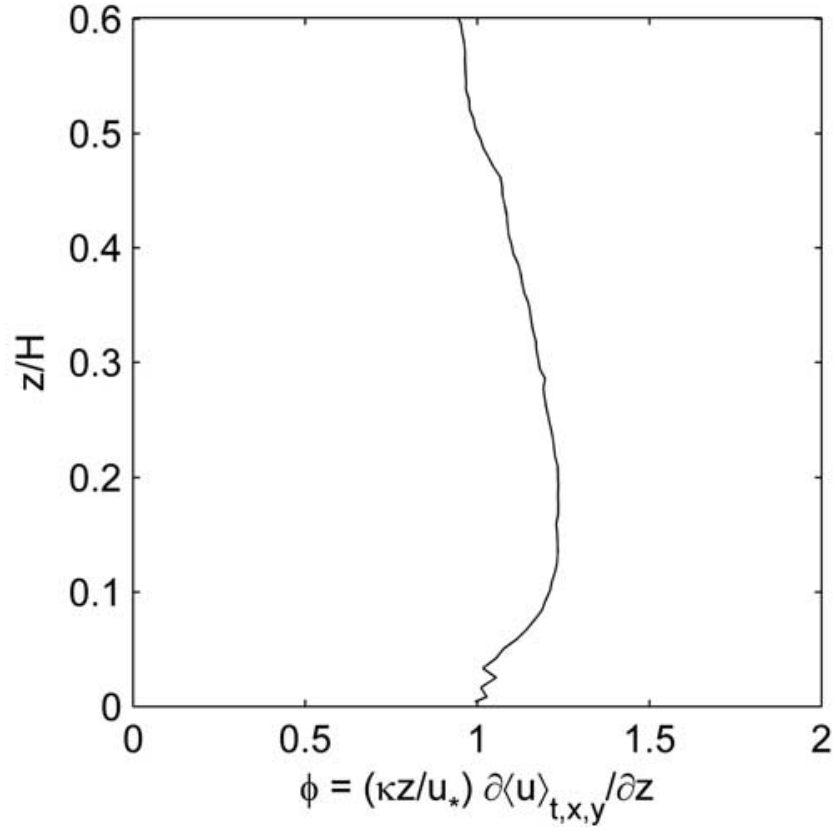

Figure 3. Vertical profile of the nondimensional gradient of the mean streamwise velocity.

gradient. The value of this gradient, being close to 1 near the surface, confirms that the velocity profile is logarithmic.

[25] Figure 4 depicts the total stress and its distribution into resolved and SGS components in dimensionless units (normalized by $u_{*}^{2}$ ). Note how the average SGS stress is insignificant everywhere except close to the surface. On the other hand, the resolved stress is 0 at the surface where all the eddies associated with momentum transfer (stress) are unresolved. The stress distributions are in qualitative agree-

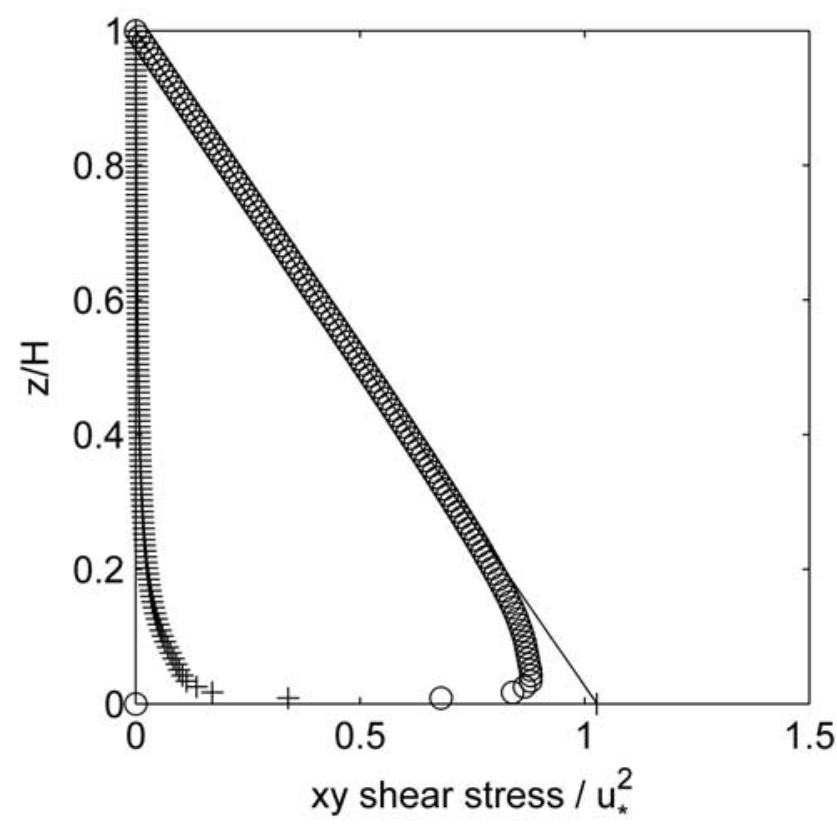

Figure 4. Vertical profiles of the resolved stress $-\left\langle\tilde{u}^{\prime} \tilde{w}^{\prime}\right\rangle_{\mathrm{t}, \mathrm{y}, \mathrm{x}}$ (circles), the subgrid-scale stress $-\left\langle\tau_{\mathbf{x z}}\right\rangle_{\mathrm{t}, \mathrm{y}, \mathrm{x}}$ (crosses), and the total stress (solid line). 


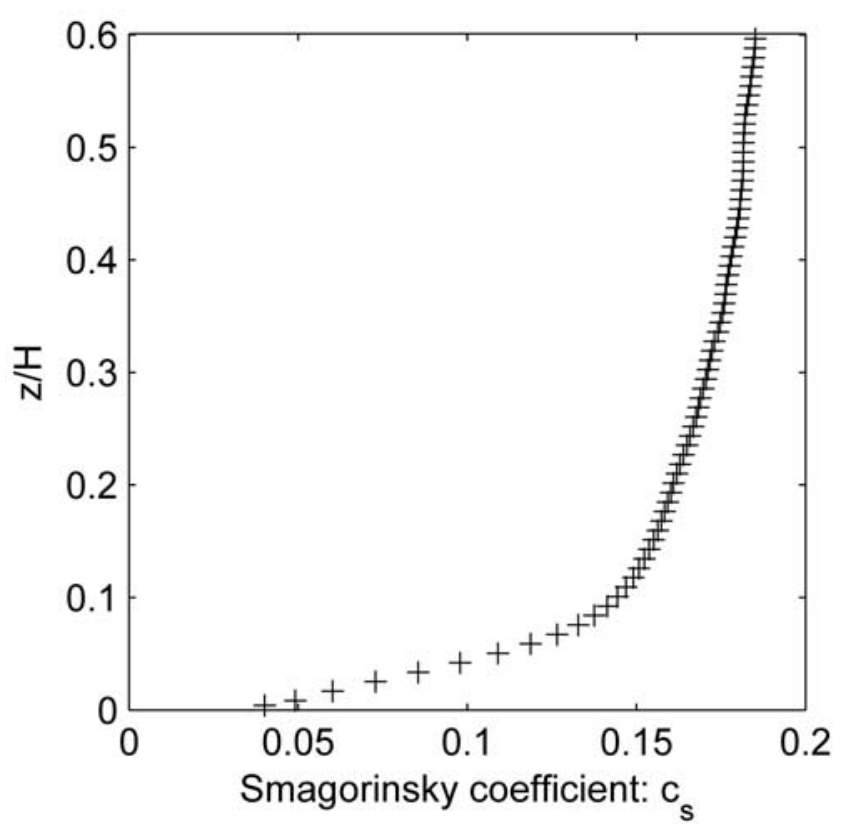

Figure 5. Vertical profile of the dynamic coefficient $\left\langle c_{s}\right\rangle_{t, x, y}$ computed by the Lagrangian-averaged scale-dependent dynamic SGS model.

ment with several LES studies (see the comparative study of Andren et al. [1994]). However, the partitioning of stress into resolved and SGS parts depends on the SGS model and the resolution of the simulation.

[26] Figure 5 presents the dynamically obtained coefficient $\left(c_{s}\right)$. The coefficient has a value of about 0.18 at the middle of the domain and decreases near the surface, which agrees well with the wall damping trend expected using the dynamic formulation. Figure 6 confirms that the vertical

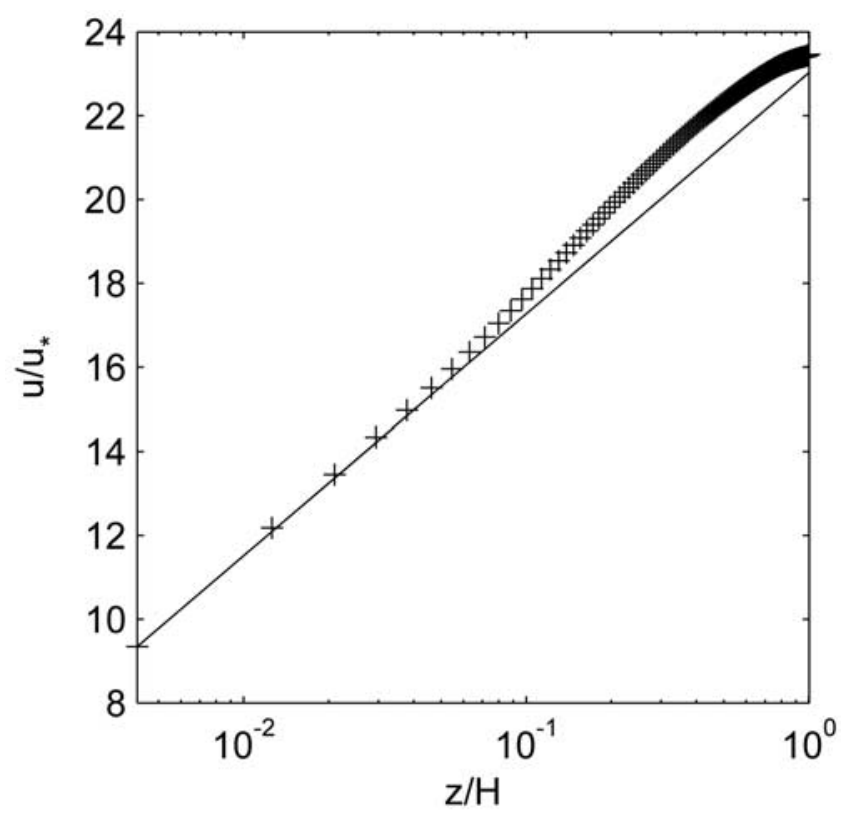

Figure 6. Logarithmic velocity profile $\langle u\rangle_{\mathrm{t}, \mathrm{x}, \mathrm{y}} / \mathrm{u} *$ (pluses) and theoretical profile $\langle u\rangle / u_{*}=(1 / \kappa) \ln \left(z / z_{\mathrm{o}}\right)$ with $\kappa=0.4$ and $z_{o}=0.1 \mathrm{~m}$ (solid line).

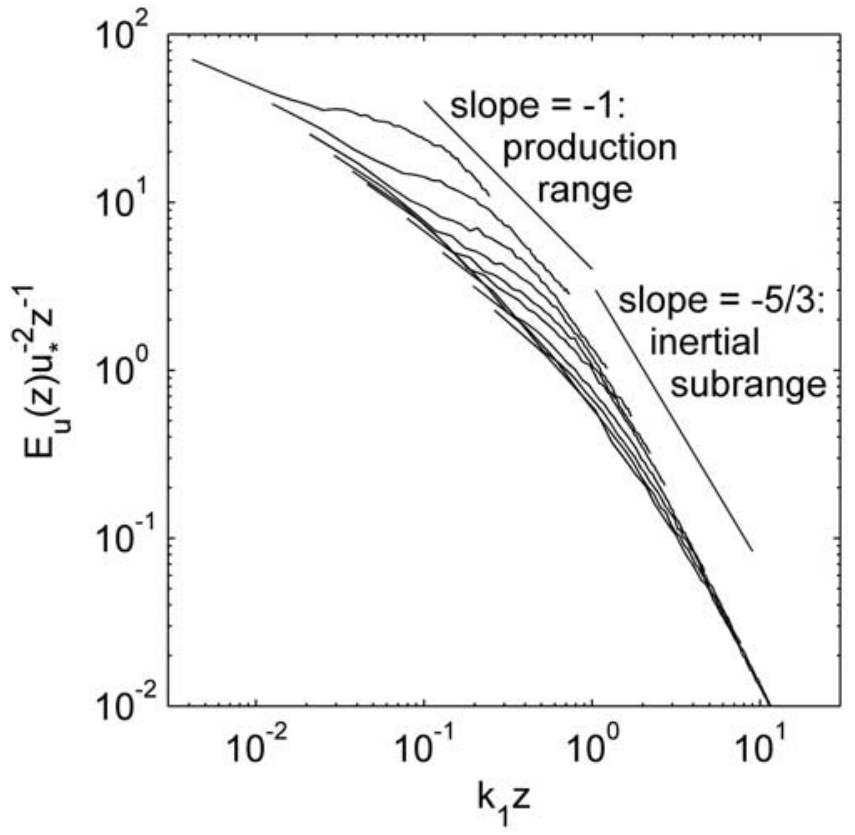

Figure 7. Normalized $u$ velocity spectra versus $k_{1} z ; k_{1}$ is the streamwise wave number and $z$ is the height. Each line represents the data for a selected height (at 4.2, 12.6, 21, 29, $38,46,80130,197$, or $267 \mathrm{~m}$ ).

profile of the $u$ velocity (streamwise) is logarithmic near the ground and departs from the logarithmic distribution further up.

[27] Figure 7 depicts the spectra of the streamwise velocity. In the inertial subrange $\left(k_{1} z>1\right)$, the effects of viscosity, boundary conditions, and large-scale structures are not important and the turbulence is essentially isotropic. The energy cascade in this subrange follows the Kolmogorov spectrum yielding a slope of $-5 / 3$. In the production range $\left(k_{1} z<1\right)$, energy cascade is affected by the flow configuration. In wall-bounded flows with neutral stability, the energy spectrum in the production range has often been shown to follow a slope of -1 from scaling arguments and experimental results [Perry et al., 1987; Katul et al., 1995]. The slopes of -1 and $-5 / 3$ are well reproduced by the LES data. As shown by Porté-Agel et al. [2000], simulation results using other SGS models depict either higher slopes (traditional Smagorinsky model) indicating over-dissipation, or lower slopes (scale-invariant formulations) indicating under-dissipation of energy. The departure from expected slopes for the alternative SGS models was especially noticeable close to the wall.

\section{Stress and Velocity Variations Over Heterogeneous Surfaces}

[28] This section presents the basic results of the ABL flow over heterogeneous surfaces. Close to the surface, the fluid is expected to move faster over the low roughness patch than over the high roughness patch. Therefore the fast moving fluid from the low roughness patch causes a high shearing stress at the leading edge of the high roughness patch that exceeds the equilibrium stress over that patch. This high shear decelerates the fluid and the stress eventu- 

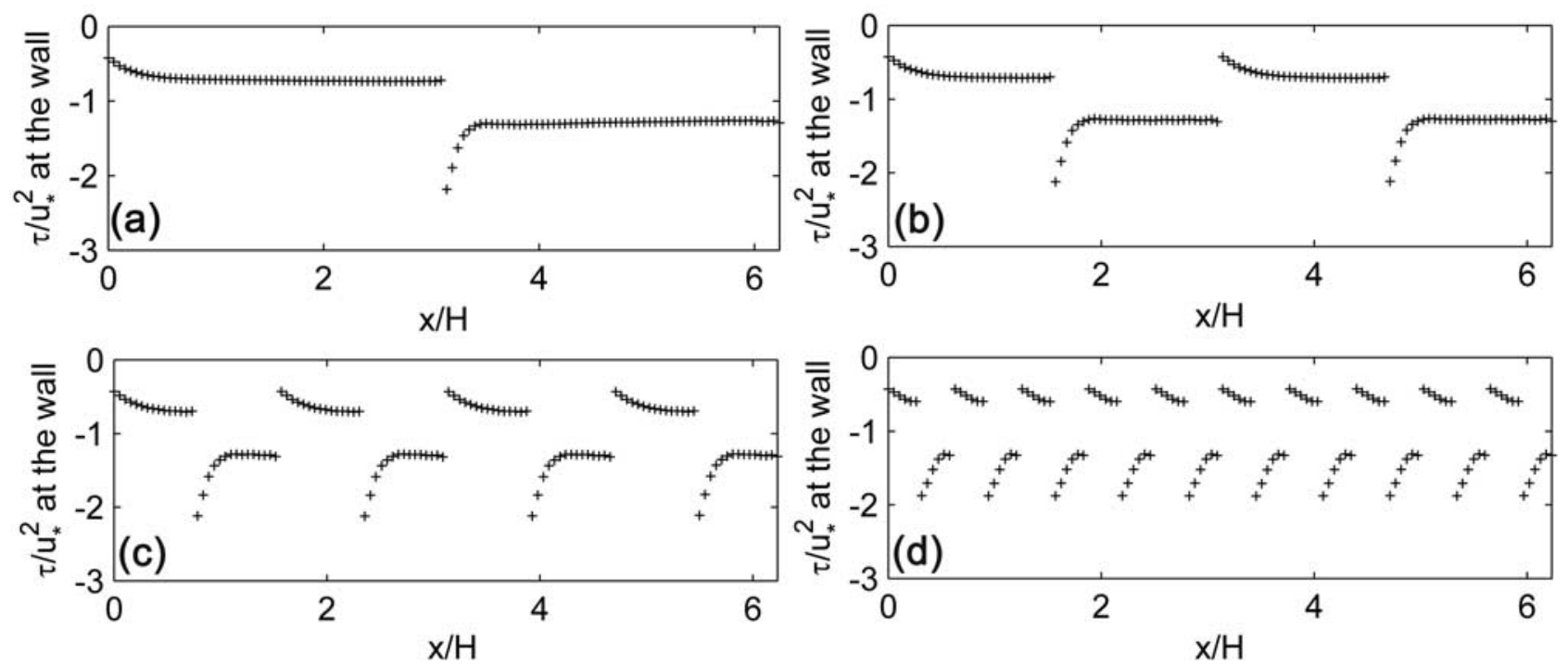

Figure 8. Nondimensional surface shear stress $\left(\left\langle\tau_{\mathrm{xz}}\right\rangle_{\mathrm{y}, \mathrm{t}} / \mathrm{u}_{*}^{2}\right)$ versus streamwise distance for different patch lengths. (a) Simulation A2, (b) simulation A4, (c) simulation A8, and (d) simulation A20.

ally decreases to its equilibrium value, if the high roughness patch is long enough to allow that. Conversely, the slow moving fluid causes a low stress over the leading edge of the low roughness patch. The fluid then accelerates and the stress increases to its equilibrium value over that patch. These overshoots and undershoots of surface stress were observed in experimental studies [Bradley, 1968; Antonia and Luxton, 1971, 1972] and in numerical simulations [Albertson and Parlange, 1999b; Glendening and Lin, 2002]. Figure 8 indicates the presence of the overshoots and undershoots in the present LES results. Figure 8 depicts the nondimensional surface shear stress from simulations A2, A4, A8, and A20. Note that the low-to-high roughness transition introduces a larger departure from equilibrium $\left(\sim u_{*}^{2}\right)$ compared with the high-to-low roughness transition $\left(\sim 0.25 u_{*}^{2}\right)$. However, the high roughness patch equilibrates faster. Figure 8 suggests that equilibrium over the low roughness patch occurs at about $0.75 \mathrm{H}$ downstream of the jump (where $H$ is the ABL depth), compared to $0.25 H$ for the high roughness patch. For cases with patch sizes smaller than the equilibrium distance, equilibrium is of course not reached. The analysis for short patches is complicated by the fact that the flow coming from the upstream patch also might not be in equilibrium. For example, note that the jump in stress at the low-to-high roughness transition for the 20-patch case is smaller than the jump for the 2-patch case. This is due to lower upstream velocities and stresses at the transition point for the 20-patch case.

[29] Figure 9 presents the streamwise velocity data at the plane of nodes closest to the surface $(d z / 2=4.2 \mathrm{~m}$ above the surface) from simulations A2, A4, A8, and A20. Acceleration of the flow over the low roughness patch and
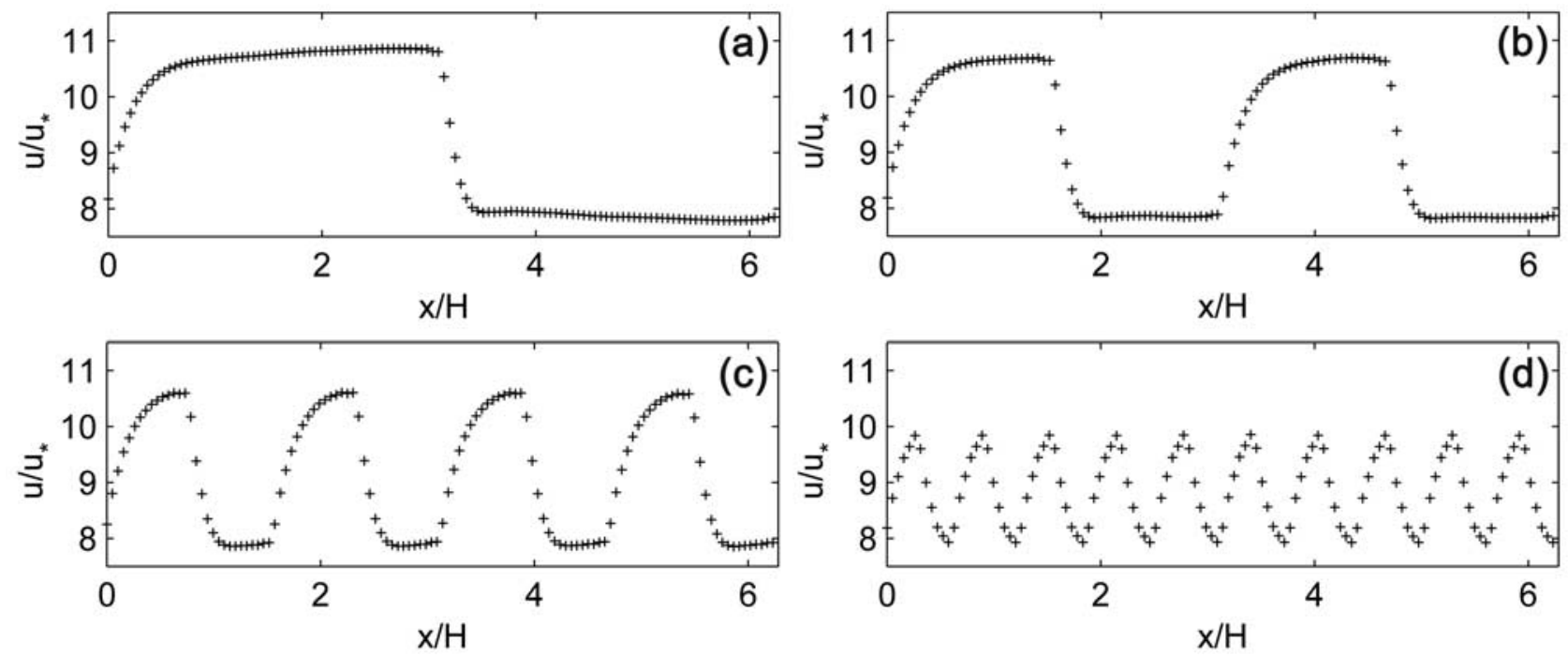

Figure 9. Nondimensional streamwise velocity $\left(\langle u\rangle_{\mathrm{y}, \mathrm{t}} u_{*}\right)$ at the first plane above the surface $(z=4.2 \mathrm{~m})$ for different patch lengths. (a) Simulation A2, (b) simulation A4, (c) simulation A8, and (d) simulation A20. 


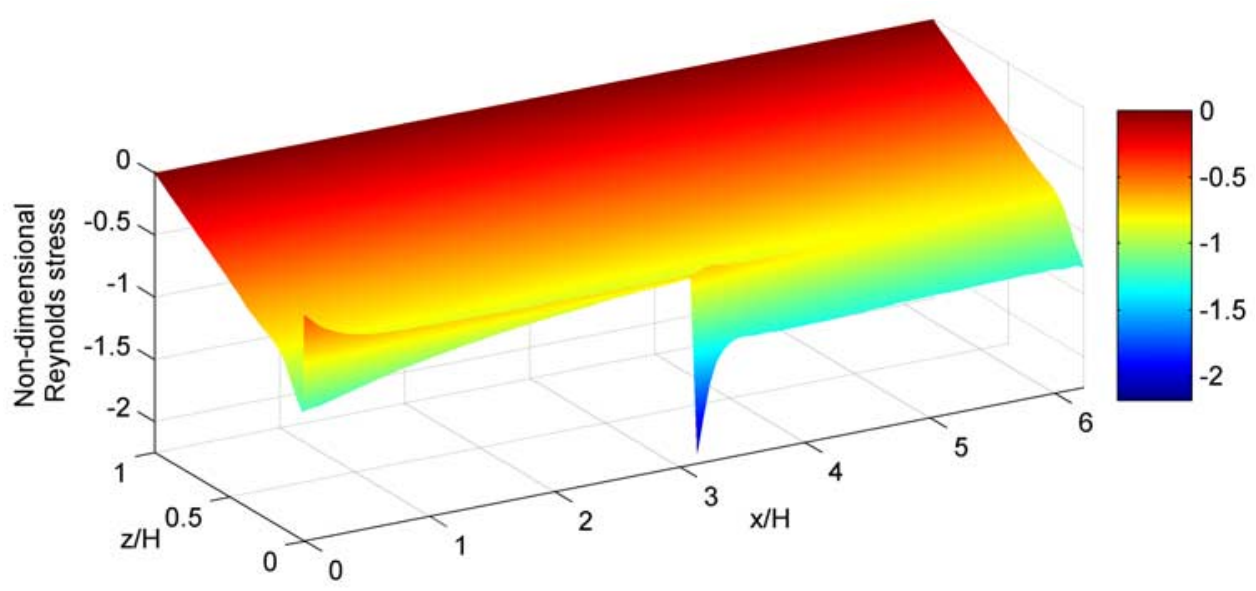

Figure 10. Reynolds stress variation over the $\mathrm{x}-\mathrm{z}$ plane $-\left\langle\tilde{u}^{\prime} \tilde{\mathrm{w}}^{\prime}\right\rangle_{t, y} / u *^{2}$. The first patch has a low roughness and is characterized by a low stress; the second patch has a higher roughness with a higher stress. The variations blend out as $z / H$ increases: 2-patch (A2) case.

deceleration over the high roughness patch are in agreement with the trends seen in the surface stress data of Figure 8. However, the distances where equilibrium is reached are significantly greater than for stress equilibrium. Hence the velocity does not reach its equilibrium value at the end of each patch for the 8-patch and 20-patch cases.

[30] Figure 10 is a plot of the resolved Reynolds stress over the $\mathrm{x}-\mathrm{z}$ plane. In agreement with Albertson and Parlange [1999b], it shows variations over patches with different roughness and the blending out of these variations with height. The nondimensional vertical velocity $(\langle w\rangle / u *)$ is depicted in Figure 11. The high-to-low roughness jump produces flow acceleration in the $\mathrm{x}$-direction resulting in downward vertical velocities. The opposite occurs after a low-to-high roughness transition resulting in an upward mass flux. This upward flux, in conjunction with the increased turbulent shearing over the high roughness patch, is important for scalar flux estimation. These conditions will increase the ability of the flow to remove heat or moisture from the surface and transport them upward in the ABL.

\section{Blending Height Determination From LES Data}

[31] With an infinite succession of patches, the flow near the wall forms an internal boundary layer (IBL) and is mainly controlled by the underlying surface. Further up, the flow is affected by "plumes" of upstream patches and is controlled by the characteristics of the upstream surface. Above the blending height $\left(h_{\mathrm{b}}\right)$, the flow is homogeneous. These trends can be visualized by plotting the deviation of the local mean velocity gradient in the vertical direction from its value averaged over all patches; i.e., by plotting $\partial\langle u\rangle_{\mathrm{t}, \mathrm{y}} / \partial z-\partial\langle u\rangle_{\mathrm{t}, \mathrm{x}, \mathrm{y}} / \partial z$ (Figure 12).

[32] The blending height $\left(h_{\mathrm{b}}\right)$, can be determined from streamwise velocity data averaged in time and in the crossstream direction. To obtain a quantitatively robust method, deviations from the $\mathrm{x}$-averaged velocity, $\langle u\rangle_{\mathrm{t}, \mathrm{y}}-\langle u\rangle_{\mathrm{t}, \mathrm{x}, \mathrm{y}}$, will be used. Figure 13 shows some selected profiles of $\langle u\rangle_{\mathrm{t}, \mathrm{y}}-\langle u\rangle_{\mathrm{t}, \mathrm{x}, \mathrm{y}}$ for simulation A2 with 2 patches. As can be seen, the profiles congregate and are very close to 0 above some blending height.

[33] At each height, the lower and upper quartiles of all the profiles are determined and plotted versus height. This results in 2 vertical profiles as depicted in the four representative examples of Figure 14. Blending is indicated by the "neck" in the profile (see the blow up boxes and horizontal lines). This gives an unambiguous indication of the point above which the quartiles no longer oscillate significantly with height indicating a fairly homogeneous flow.

[34] Figure 15 is a plot of the blending height determined by the above mentioned method versus patch length, for all

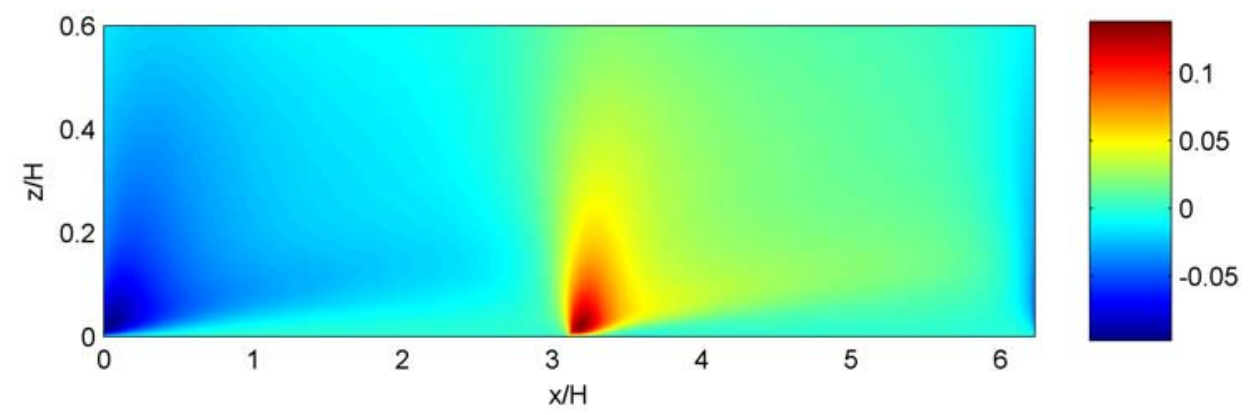

Figure 11. Nondimensional vertical velocity $\left(\langle w\rangle_{\mathrm{t}, \mathrm{y}} / u_{*}\right)$ : 2-patch (simulation A2) case. 


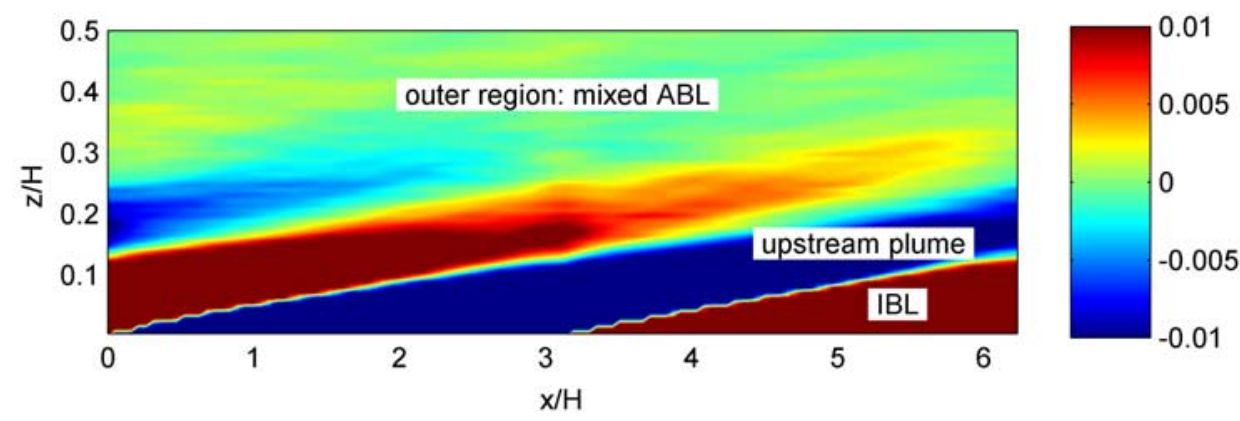

Figure 12. Plot of $\left[\partial\langle u\rangle_{\mathrm{t}, \mathrm{y}} / \partial z-\partial\langle u\rangle_{\mathrm{t}, \mathrm{x}, \mathrm{y}} / \partial z\right] H u^{*}$ depicting the different layers in the ABL above patchy surfaces.

scenarios. Clearly $h_{\mathrm{b}}$ grows with patch length. In addition, the surface roughness has a noticeable effect upon the magnitude of $h_{\mathrm{b}}$.

\section{Effective Surface Roughness}

[35] Most attempts to apply the law-of-the-wall or MOS to heterogeneous surfaces use the concept of "equivalent surface roughness" $z_{o, e}$. This equivalent roughness should, when used in the similarity law expressions, reproduce the average velocity or average stress profiles observed over heterogeneous surfaces. A concern regarding this concept is that the value of $z_{o, e}$ that reproduces the stress is different from the value reproducing velocity [Taylor, 1987; Mason, 1988]. In addition, one has to know the proper parameters (velocity, von Karman constant, or stress) to use in the similarity equations. Since such an approach is mostly used in mesoscale modeling of ABL flows to impose a wall stress, it is the view of the authors that it is most important to have a $z_{o, e}$ that predicts the correct surface stress, given velocity data from the simulation.

[36] In LES, the surface is assumed homogeneous at the grid scale. At larger scales the equivalent surface roughness for the whole modeling domain can be estimated from averaged velocity data by assuming a log-law of the form

$$
\frac{\langle u\rangle_{\mathrm{t}, \mathrm{x}, \mathrm{y}}}{u *}=\frac{1}{\kappa} \ln \left(\frac{z}{z_{\mathrm{o}, \mathrm{e}}}\right) .
$$

[37] A least squares error fit between the measured $\langle u\rangle_{\mathrm{t}, \mathrm{x}, \mathrm{y}} / u *$ and the law-of-the-wall expression leads to the following equation

$$
\ln \left(z_{\mathrm{o}, \mathrm{e}}\right)=\frac{1}{n_{\mathrm{p}}}\left[\sum_{\mathrm{i}=1}^{n_{\mathrm{p}}} \ln \left(z_{\mathrm{i}}\right)-\kappa\left(\left\langle u\left(z_{\mathrm{i}}\right)\right\rangle_{\mathrm{t}, \mathrm{x}, \mathrm{y}} / u *\right)\right],
$$

where $n_{\mathrm{p}}$ is the number of data points along the z-axis used in the data fit; the first 6 data points $\left(n_{\mathrm{p}}=6\right)$ above the wall were used. This procedure is equivalent to fitting a straight line through the average nondimensional velocity profile $\left(\langle u\rangle_{\mathrm{t}, \mathrm{x}, \mathrm{y}} / u_{*}\right)$ plotted versus the logarithm of elevation (similar to Figure 6). The slope of the line is imposed as $1 / \kappa$; the intercept of that line with the zero-velocity axis gives the equivalent roughness height $z_{\mathrm{o}, \mathrm{e}}$. Figure 16 depicts four examples where the velocity profile was used to get $z_{\mathrm{o}, \mathrm{e}}$. The blending heights and effective surface roughness values determined from LES data are given in Table 3.

\section{Internal Boundary Layer Growth}

[38] The aim of this section is to understand the blending height behavior from the evolution of internal boundary layers (IBLs) emanating from the boundaries of the patches. Several methods of identifying the IBLs exist (see Appen$\operatorname{dix} A$ ). It is useful in this work to determine the IBL height $\left(\delta_{\mathrm{IBL}}\right)$ based on velocity data as the point where $\partial\langle u\rangle_{\mathrm{t}, \mathrm{y}} / \partial z=$ $\partial\langle u\rangle_{\mathrm{t}, \mathrm{x}, \mathrm{y}} / \partial z$. This corresponds to the point where the velocity profile has become insensitive to the surface directly underneath. However, the flow has not blended yet; the velocity is mainly controlled by the flow from the upstream patch (Figure 12). The $\delta_{I B L}$ determined using this method is depicted in Figure 17 for simulation (A2). The results did not reveal significant differences between low-to-high and high-to-low roughness transitions. This is in agreement with experimental results [Bradley, 1968] and numerical results [Rao et al., 1973, Glendening and Lin, 2002]. This is valid

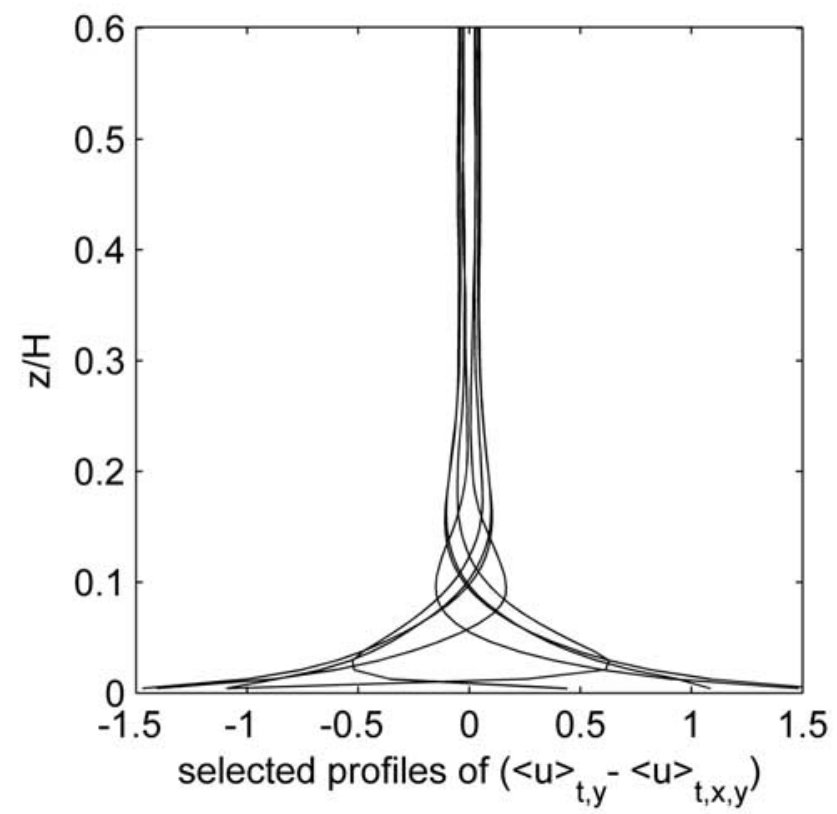

Figure 13. Selected profiles of $\langle u\rangle_{t, y}-\langle u\rangle_{t, x, y}$ at different $x$, normalized by $u^{*}$. 

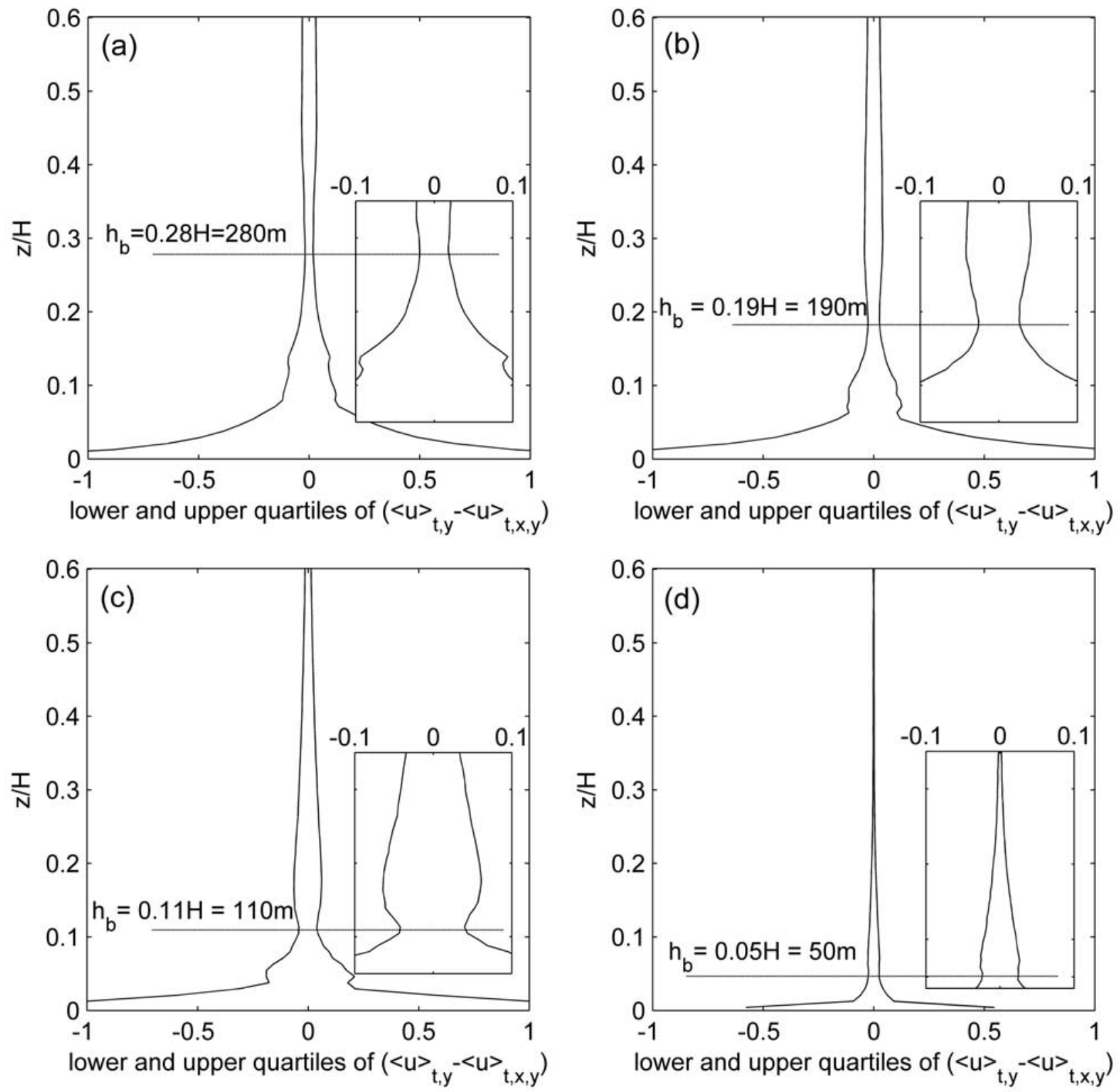

Figure 14. Examples of profiles of lower and upper quartiles of the set of profiles $\langle u\rangle_{\mathrm{t}, \mathrm{y}}-\langle u\rangle_{\mathrm{t}, \mathrm{x}, \mathrm{y}}$. Zoom-in area depicts the "neck" of the profile corresponding to the blending height. The determined blending height is indicated by the dashed line. (a) Simulation A2, (b) simulation B4, (c) simulation C8, and (d) simulation D20.

only if the transition is between two rough patches. The IBL will be sensitive to the type of transition if the lowroughness patch approaches the limit where it becomes "hydrodynamically" smooth [Antonia and Luxton, 1971, 1972] and might be sensitive to the type of transition if the roughness ratio $z_{\mathrm{o}, 2} / z_{\mathrm{o}, 1}$ is significantly higher than the ratios used in this work (about 100).

[39] $\delta_{\text {IBL }}$ determined for other simulations (with different surface roughness and different patch scales) reveal similar characteristics. Furthermore, the IBL growth was not significantly affected by the patch length, unless the patch scale variations affect the effective surface roughness. This suggests that the effective surface roughness is the main parameter controlling the land-atmosphere interaction; the
$\mathrm{ABL}$ is responding similarly to the increase and decrease in surface roughness. Hence a parameterization for the blending height might be obtained using the effective surface roughness and the patch length.

[40] To better understand the physical mechanisms controlling the growth of the IBL, a classic scaling argument is revisited. This widely used approach to estimate the growth of the boundary layer applies diffusion analogy (introduced by Miyake [1965]; see Garratt [1990] for a review of the proposed modifications and verification of the approach). The scaling arguments used are the following:

$$
\frac{d \delta_{\mathrm{IBL}}}{d x} \sim \frac{\text { upward diffusion velocity }}{\text { streamwise convection velocity }} \sim \frac{w_{\mathrm{rms}}}{\left\langle u\left(\delta_{\mathrm{IBL}}\right)\right\rangle},
$$




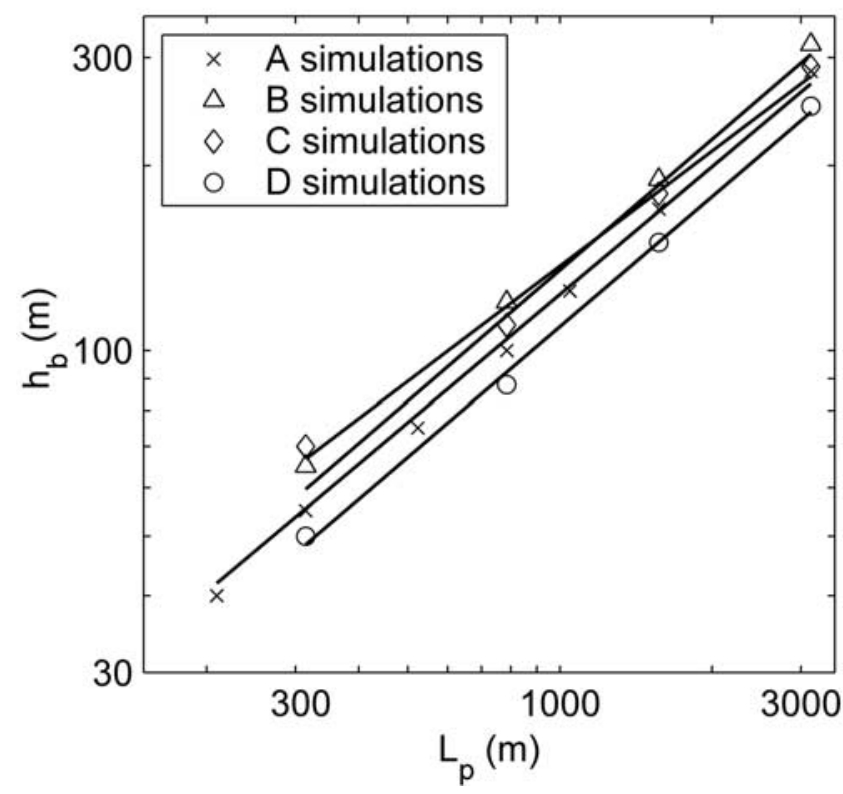

Figure 15. Blending height $\left(h_{\mathrm{b}}\right)$ versus patch length $\left(L_{\mathrm{p}}\right)$ for all simulations; solid lines are power law fits.

where $\mathrm{x}$ is the downstream distance, $\delta_{I B L}$ the IBL depth, $\left\langle u\left(\delta_{\mathrm{IBL}}\right)\right\rangle$ the mean streamwise velocity at the IBL top, and $w_{\text {rms }}$ the standard deviation (the root mean square) of the vertical velocity. $w_{\mathrm{rms}}$ can be approximated by $u_{*}$ (from LES data, for the lower $20 \%$ of the ABL, $\left.w_{\text {rms }} \sim 0.95 u_{*}\right)$ whereas the mean streamwise velocity can be estimated from an effective log profile. Hence

$$
\frac{d \delta_{\mathrm{IBL}}}{d x}=C \frac{u_{*}}{\left\langle u\left(\delta_{\mathrm{IBL}}\right)\right\rangle}=C \frac{\kappa}{\ln \left(\delta_{\mathrm{IBL}} / z_{\mathrm{o}, \mathrm{e}}\right)} .
$$

$C$ is an adjustable proportionality constant, $u_{*}$ the friction velocity, $z_{o, e}$ the effective surface roughness, and $\kappa$ the vonKarman constant. Rearranging the equation, we obtain

$$
\ln \left(\delta_{\mathrm{IBL}} / z_{\mathrm{o}, \mathrm{e}}\right) d \delta_{\mathrm{IBL}}=C \kappa d x
$$

Table 3. Summary of the $h_{\mathrm{b}}$ and $z_{\mathrm{o}, \mathrm{e}}$ Values Determined From LES Results for All Simulations

\begin{tabular}{ccc}
\hline Simulation & $h_{\mathrm{b}}, \mathrm{m}$ & $z_{\mathrm{o}, \mathrm{e}}, \mathrm{m}$ \\
\hline A1 & $\mathrm{n} / \mathrm{a}$ & 0.101 \\
A2 & 285 & 0.104 \\
A4 & 170 & 0.105 \\
A6 & 125 & 0.104 \\
A8 & 100 & 0.108 \\
A12 & 75 & 0.115 \\
A20 & 55 & 0.123 \\
A30 & 40 & 0.130 \\
B2 & 315 & 0.366 \\
B4 & 190 & 0.372 \\
B8 & 120 & 0.402 \\
B20 & 65 & 0.459 \\
C2 & 290 & 0.229 \\
C4 & 180 & 0.262 \\
C8 & 110 & 0.284 \\
C20 & 70 & 0.373 \\
D2 & 250 & 0.0255 \\
D4 & 150 & 0.0256 \\
D8 & 88 & 0.0300 \\
D20 & 50 & 0.0339 \\
\hline
\end{tabular}

Integrating from $\left(\delta_{\mathrm{IBL}}=z_{\mathrm{o}, \mathrm{e}}\right.$ at $\left.\mathrm{x}=0\right)$ to $\left(\delta_{\mathrm{IBL}}\right.$ at $\left.\mathrm{x}\right)$ yields

$$
\begin{gathered}
\int_{z_{\mathrm{o}, \mathrm{e}}}^{\delta_{\mathrm{IBL}}} \ln \left(\delta_{\mathrm{IBL}} / z_{\mathrm{o}, \mathrm{e}}\right) d \delta_{\mathrm{IBL}}=\int_{0}^{x} C \kappa d x, \text { or } \\
\left(z_{\mathrm{o}, \mathrm{e}}-\delta_{\mathrm{IBL}}\right)+\delta_{\mathrm{IBL}} \ln \left(\delta_{\mathrm{IBL}} / z_{\mathrm{o}, \mathrm{e}}\right)=C \kappa x,
\end{gathered}
$$

which can be rearranged to get

$$
\frac{\delta_{\mathrm{IBL}}}{z_{\mathrm{o}, \mathrm{e}}}\left[\ln \left(\frac{\delta_{\mathrm{IBL}}}{z_{\mathrm{o}, \mathrm{e}}}\right)-1\right]+1=C \kappa \frac{x}{z_{\mathrm{o}, \mathrm{e}}} .
$$

The +1 is insignificant since typically $\delta_{\mathrm{IBL}} / \mathrm{z}_{\mathrm{o}, \mathrm{e}} \gg 1$ and the equation can be written as

$$
\delta_{\mathrm{IBL}}\left[\ln \left(\frac{\delta_{\mathrm{IBL}}}{z_{\mathrm{o}, \mathrm{e}}}\right)-1\right]=C \kappa x \text {. }
$$

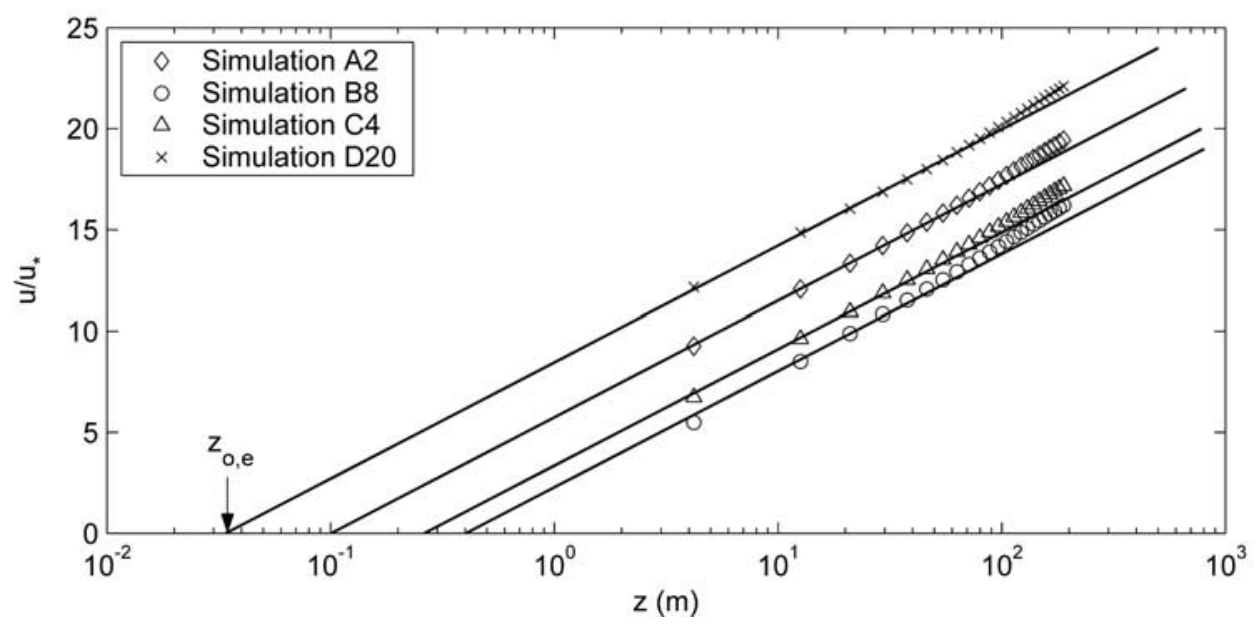

Figure 16. Velocity profiles $\left(\langle u\rangle_{\mathrm{t}, \mathrm{x}, \mathrm{y}} u_{*}\right)$ for four simulations; the straight lines are the least squares fits to the first six points. The intercept with the axis of $u / u_{*}=0$ gives $z_{\mathrm{o}, \mathrm{e}}$. 


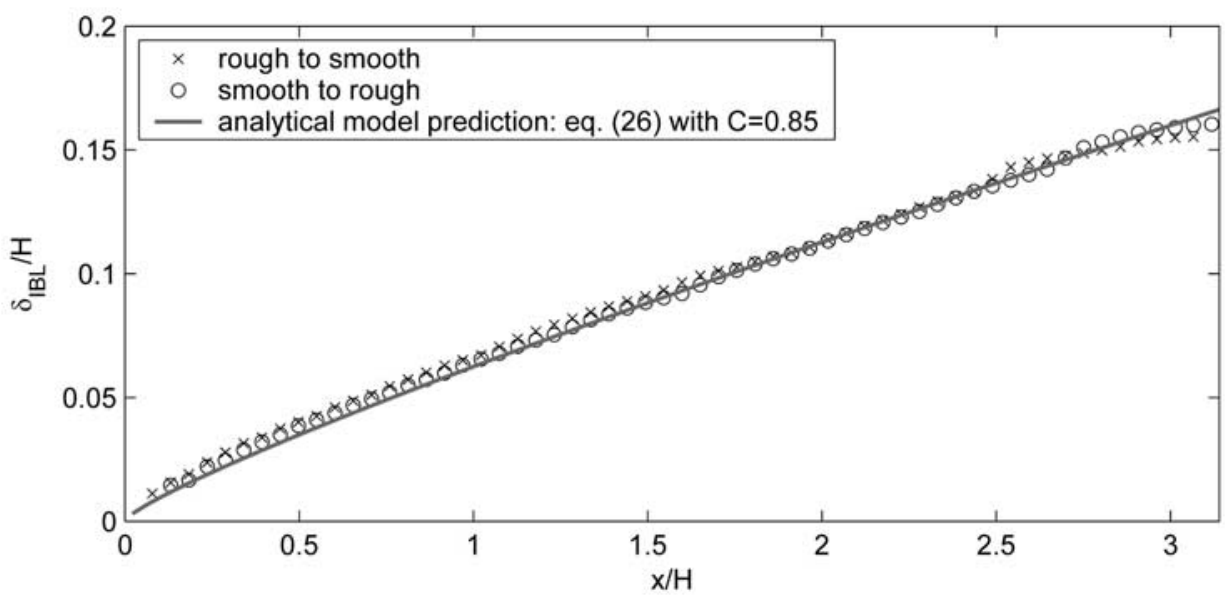

Figure 17. IBL growth after a jump in surface roughness for simulation A2.

[41] As shown in Figure 17, good agreement is obtained with LES results after choosing a coefficient of $C=0.85$. The IBL thicknesses from all the 2-patch simulations were measured from LES and compared to the prediction of equation (26) with the measured equivalent surface roughness for each simulation. Figure 18 indicates that the agreement is quite good using the same value of $C=0.85$ for all cases (although the B2 and C2 cases are slightly underpredicted). It should be noted that even minor changes in $z_{\mathrm{O}, \mathrm{e}}$ have a great effect on $\delta_{\mathrm{IBL}}$; however, these effects are consistent and can be well attributed to the sensitivity of the $\langle u(z)\rangle$ profile to changes in $z_{\mathrm{o}, \mathrm{e}}$.

[42] The IBL height can be determined using other methods based on velocity or stress data. These methods were tested in this work and the results are presented in Appendix A. The results show equivalent trends, up to a multiplying factor, so that the value of the coefficient $C$ of equation (26) needed to predict IBL growth is different for each method.

\section{Parameterizations for Mesoscale Models}

\subsection{Blending Height}

[43] To explore the relationship between the IBL development and blending height $\left(h_{\mathrm{b}}\right)$, contour plots of the nondimensional $u$ gradient $\kappa z / u_{*}\left(d\langle u\rangle_{\mathrm{y}, \mathrm{t}} / d z\right)$ were inspected, with the IBL determined by equation (26) overlaid on these plots (only IBL of one patch is shown for clarity, remember that the IBLs of all patches grow similarly). Figure 19 depicts these overlaid plots. It is observed that, in all simulations, when the IBL is allowed to grow until a downstream distance roughly equal to twice the patch length, the blending height is reached. Hence it is empirically found from the LES data that

$$
h_{\mathrm{b}}=\delta_{\mathrm{IBL}}\left(x=\alpha L_{p}\right) \text { with } \alpha \approx 2 .
$$

Combining with equation (26) and $C=0.85$ indicates that the blending height can be estimated from

$$
h_{\mathrm{b}}\left[\ln \left(\frac{h_{\mathrm{b}}}{z_{o, e}}\right)-1\right]=0.85 \kappa\left(2 L_{\mathrm{p}}\right) .
$$

[44] To test this relation, blending heights for all simulations were computed and compared to the heights previously determined from LES velocity data (Figure 14 and Table 3). Figure 20 is a plot of blending height versus patch length (divided by the equivalent surface roughness to reduce the number of variables to $h_{\mathrm{b}} / z_{\mathrm{o}, \mathrm{e}}$ and $\left.L_{\mathrm{p}} / z_{\mathrm{o}, \mathrm{e}}\right)$. The data points are the heights determined from LES data; the

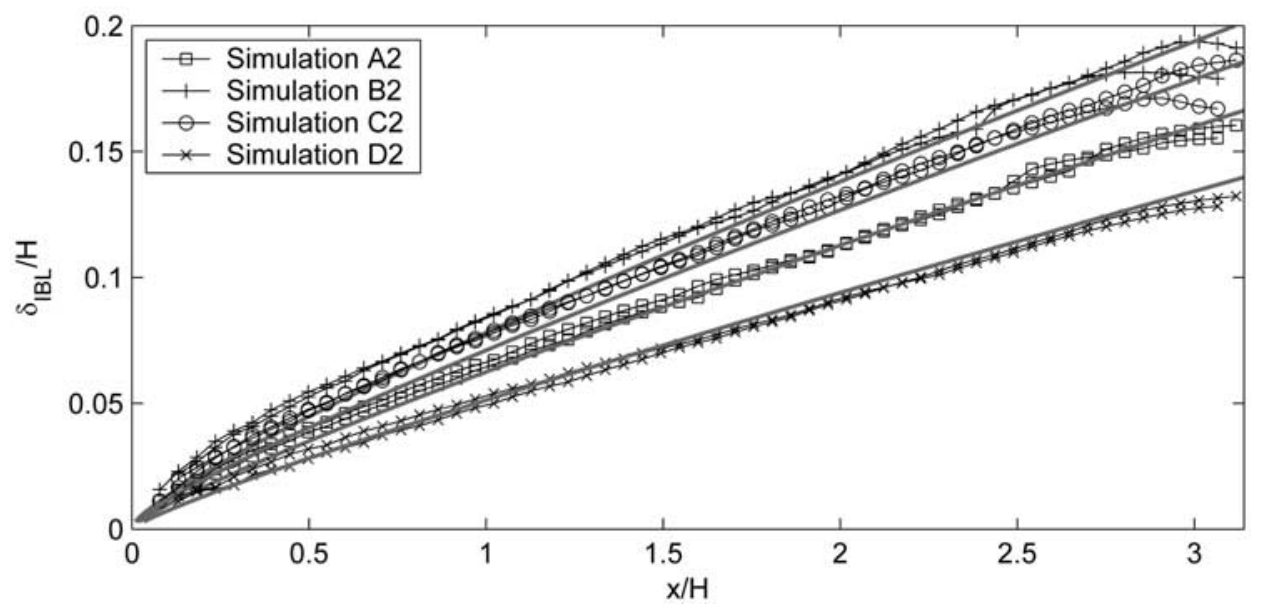

Figure 18. IBL growth for different simulations defined using $\mathrm{d}\langle u\rangle_{\mathrm{t}, \mathrm{y}} / d z=\mathrm{d}\langle u\rangle_{\mathrm{t}, \mathrm{x}, \mathrm{y}} / d z$. The shaded lines are the corresponding predictions of equation (26) with $C=0.85$, with the equivalent surface roughness determined from LES data. 

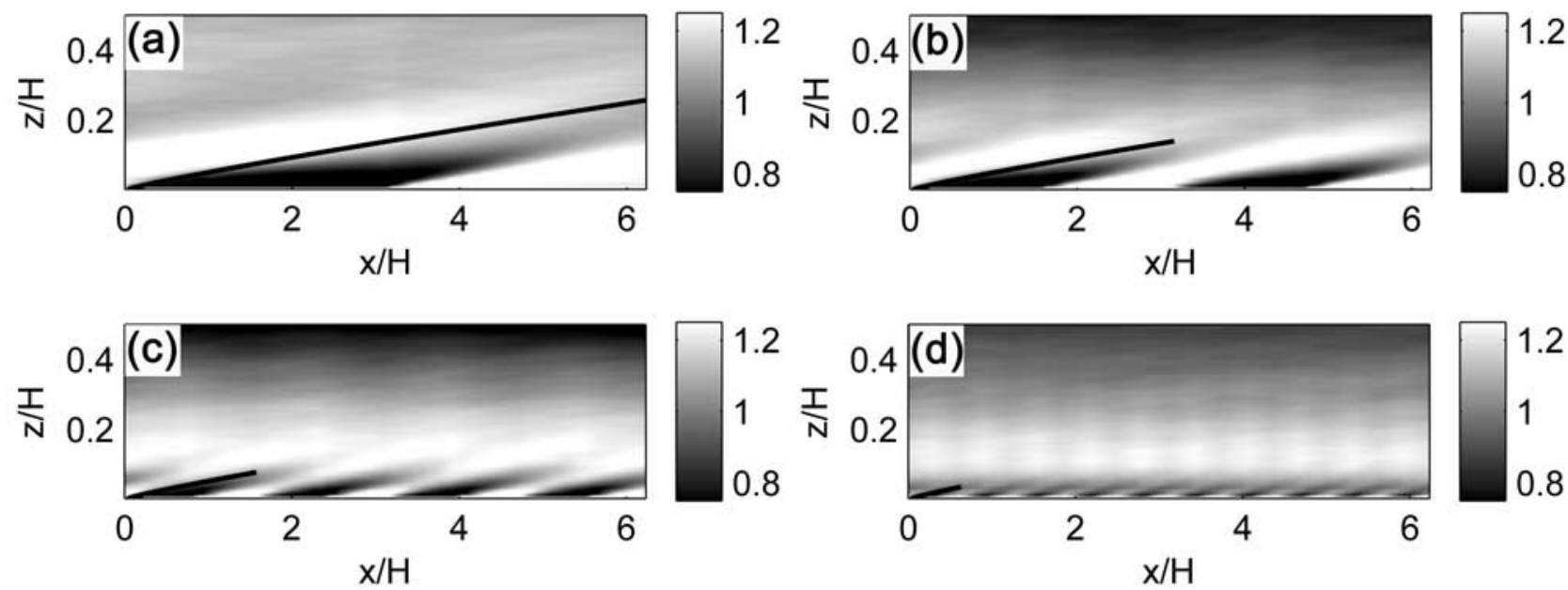

Figure 19. Plots of $k z / u_{*}\left(d\langle u\rangle_{y, t} / d z\right)$. The solid lines are the IBLs as determined from equation (26), extending over two patches. (a) Simulation A2, (b) simulation B4, (c) simulation C8, and (d) simulation D20.

solid line is the prediction of equation (28). The comparison is very satisfactory. It should be noted that the coefficient $\alpha$ could alternatively be determined from an error minimization procedure to reproduce the blending height obtained from LES data. Such an approach yields a coefficient $\alpha$ of 1.8 .

\subsection{Effective Surface Roughness}

[45] As can be noted, equation (28) used to compute the blending height involves the value of the equivalent surface roughness $z_{\mathrm{o}, \mathrm{e}}$. Up to this point, the computed equivalent roughness determined from LES data was used. Hence a parameterization for $z_{\mathrm{o}, \mathrm{e}}$ is required to allow computation of the blending height without any need for flow measurements. Moreover, as discussed in the introduction, the computation of an equivalent surface roughness is very important for mesoscale simulations.

[46] The approach presented here has been initiated by Wieringa [1986] and Mason [1988]; it was discussed and modified by Claussen [1991]. In this study, a slight mod- ification is proposed and, most importantly, the performance of the model is tested versus LES results.

[47] The total surface force over a heterogeneous area can be written as the discrete sum of surface forces over the homogeneous patches in the area

$$
A_{\text {total }} \tau_{\text {total }}=\sum_{i=1}^{N} A_{i} \tau_{i},
$$

where $N$ is the number of patches, $A_{\text {total }}$ is the total area considered, and $A_{i}$ is the area of patch $i$. Assuming that the patches are big enough so that the flow is in equilibrium with the underlying surface over most of the patch, the surface stress can be expressed using the law-of-the-wall. The equation can be rewritten as

$$
\left(\frac{u_{\mathrm{e}} \kappa}{\ln \left(\frac{z}{z_{\mathrm{o}, \mathrm{e}}}\right)}\right)^{2}=\sum_{i=1}^{N} f_{i}\left(\frac{u_{i} \kappa}{\ln \left(\frac{z}{z_{\mathrm{o}, i}}\right)}\right)^{2}
$$

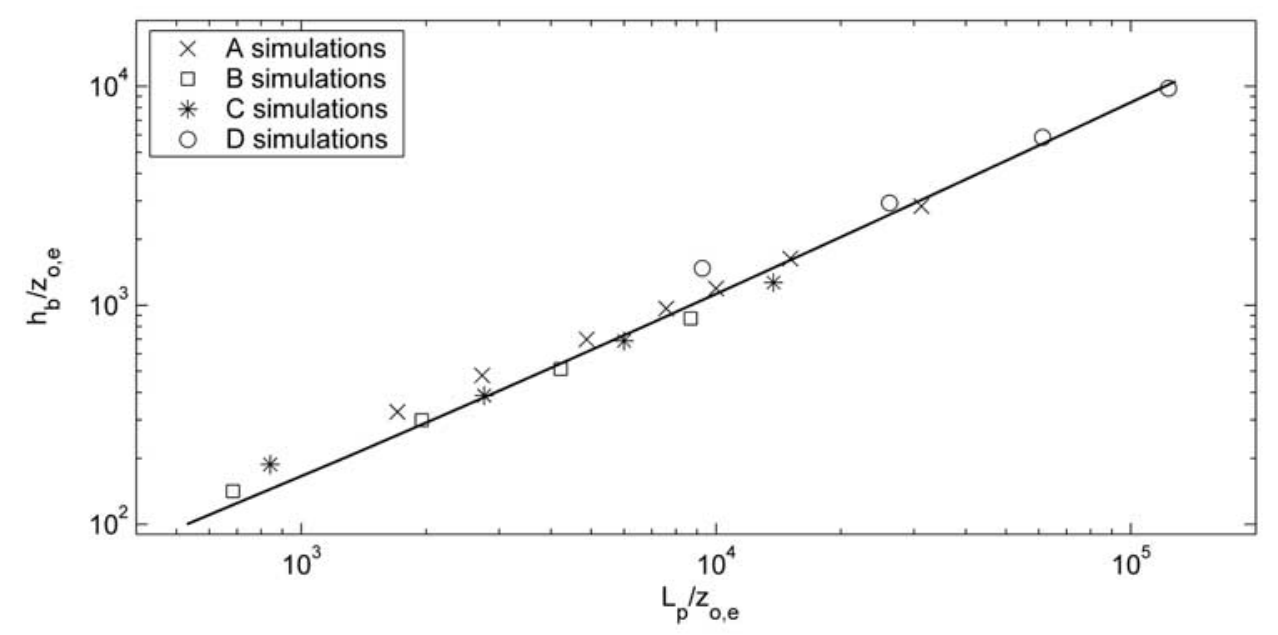

Figure 20. Normalized mixing height versus patch length for all simulations fitted with a curve of the form $h_{\mathrm{b}}\left[\ln \left(h_{\mathrm{b}} / z_{\mathrm{o}}\right)-1\right]=1.7 \kappa L_{\mathrm{p}}$. 


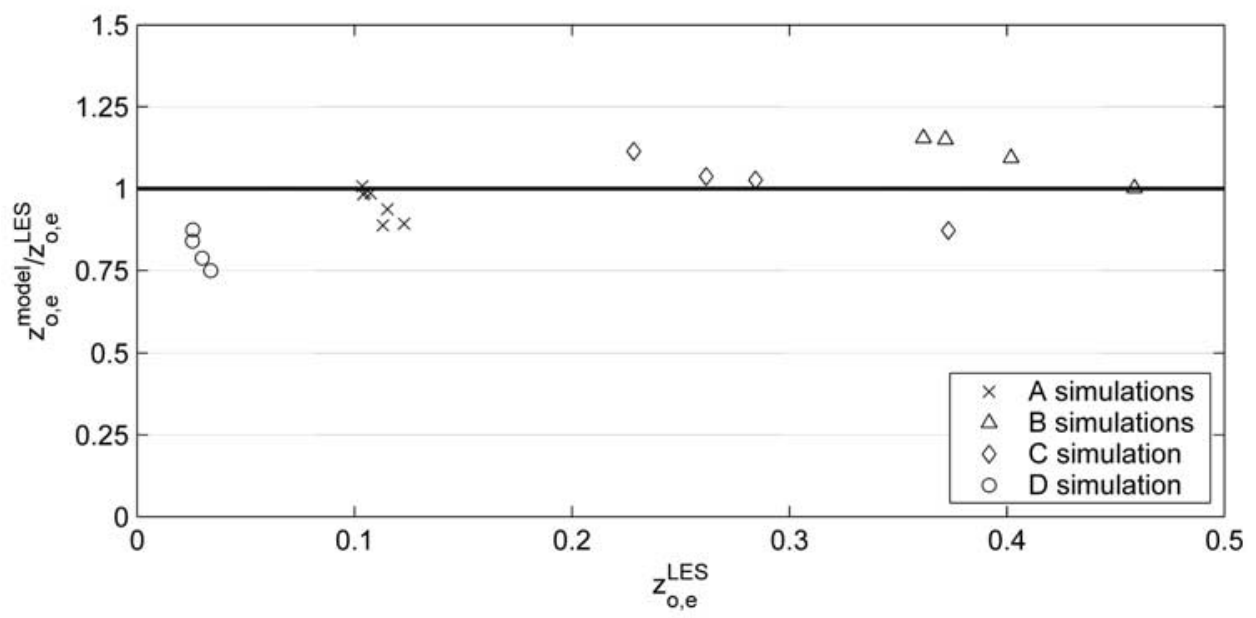

Figure 21. Comparison of equivalent surface roughness estimated by the analytical model (equations (32) and (33)) to LES values (Figure 16 and Table 3). The thick line corresponds to $z_{o}^{\text {model }}=z_{o}^{\text {LES }}$ (perfect fit).

where $u_{e}$ is the velocity averaged over the whole domain and $z_{o, e}$ is the equivalent surface roughness to be determined. Similarly, $u_{i}$ and $z_{\mathrm{o}, i}$ are the equilibrium velocity and the surface roughness of patch $i$ and $f_{i}$ is the fraction of the total area covered by patch $i\left(f_{i}=A_{i} / A_{\text {total }}\right)$. Following the approach of Wieringa [1986] and Mason [1988], the above equation is evaluated at the blending height $h_{\mathrm{b}}$ where the flow velocity is homogeneous over all the area $\left(u_{i} \approx u_{\mathrm{e}}\right.$ for any patch). Hence the equation reduces to

$$
\frac{1}{\left(\ln \frac{h_{\mathrm{b}}}{z_{\mathrm{o}, e}}\right)^{2}}=\sum_{i=1}^{N} f_{i} \frac{1}{\left(\ln \frac{h_{\mathrm{b}}}{z_{\mathrm{o}, i}}\right)^{2}} .
$$

Before solving for $z_{\mathrm{o}, \mathrm{e}}$, equations (28) and (31) can be combined to yield an equation for $h_{\mathrm{b}}$ :

$$
\left(\frac{h_{\mathrm{b}}}{1.7 \kappa L_{\mathrm{p}}+h_{\mathrm{b}}}\right)^{2}=\sum_{i=1}^{N} f_{i} \frac{1}{\left(\ln \frac{h_{\mathrm{b}}}{z_{\mathrm{o}, i}}\right)^{2}}
$$

Equation (32) needs to be solved iteratively for $h_{\mathrm{b}}$. Subsequently, the value of $z_{\mathrm{o}, \mathrm{e}}$ can be computed from a rearranged form of equation (28)

$$
z_{\mathrm{o}, e}=h_{\mathrm{b}} \exp \left[-\frac{1.7 \kappa L_{\mathrm{p}}}{h_{\mathrm{b}}}-1\right] \text {. }
$$

[48] The model presented here is used to estimate the equivalent surface roughness for the LES runs and the results are compared to the effective surface roughness determined from LES data. Figure 21 depicts the comparison results where the ratio $z_{o}^{\text {model }} / z_{o}^{\text {LES }}$ is plotted versus $z_{o}{ }^{\text {LES }}$. A value of 1 (thick black line) indicates that the model perfectly reproduces LES results. It can be noted that all model estimates are within $25 \%$ of LES values. Two other alternative formulations (reviewed in Appendix B) did not yield results as satisfactory as the model proposed here.
[49] Note that only simple configurations of patches of equal lengths were used in this work, for which the determination of the characteristic scale of heterogeneity was simple (equal to the patch length $L_{\mathrm{p}}$ ). More realistic configurations would include patches of different sizes and complex configurations. Therefore the analysis and parameterization used here are being validated for more realistic configurations. An interesting discussion of approaches that can be used to obtain a characteristic patch length can be found in a paper by Brutsaert [1998].

\section{Conclusions}

[50] A new generation large-eddy simulation (LES), based on a Lagrangian scale-dependent dynamic subgrid model, is applied to neutral atmospheric flow over heterogeneous land surfaces. A suite of simulations was performed to investigate the impact of different surface roughness values and patch lengths on the structure of the lower atmosphere. Roughness lengths were varied to represent surfaces ranging from bare soils to forests. The simulated patch lengths ranged between 200 and $3000 \mathrm{~m}$ corresponding to length scales ranging from less than the depth of the boundary layer to about 3 times the ABL depth. The magnitude of the roughness ratio was set to 10 or 100 .

[51] Validation tests showed that this LES code is faithful to the physics of the interaction of the lower atmosphere and the land surface. The validation compared LES results to well-established characteristics of ABL flows. The velocity profile was indeed logarithmic and the streamwise velocity spectra were in accordance with experimental and theoretical studies. The profile of the dynamic Smagorinsky coefficient also displayed the expected trend, starting at 0.18 away from the wall and decreasing as the ground is approached. The velocities and stresses close to the ground were examined; the trends observed at the roughness jumps reproduced several features (such as stress overshoots and undershoots) observed in experimental and numerical studies.

[52] The blending height and equivalent surface roughness were determined for all simulations. A formulation to 


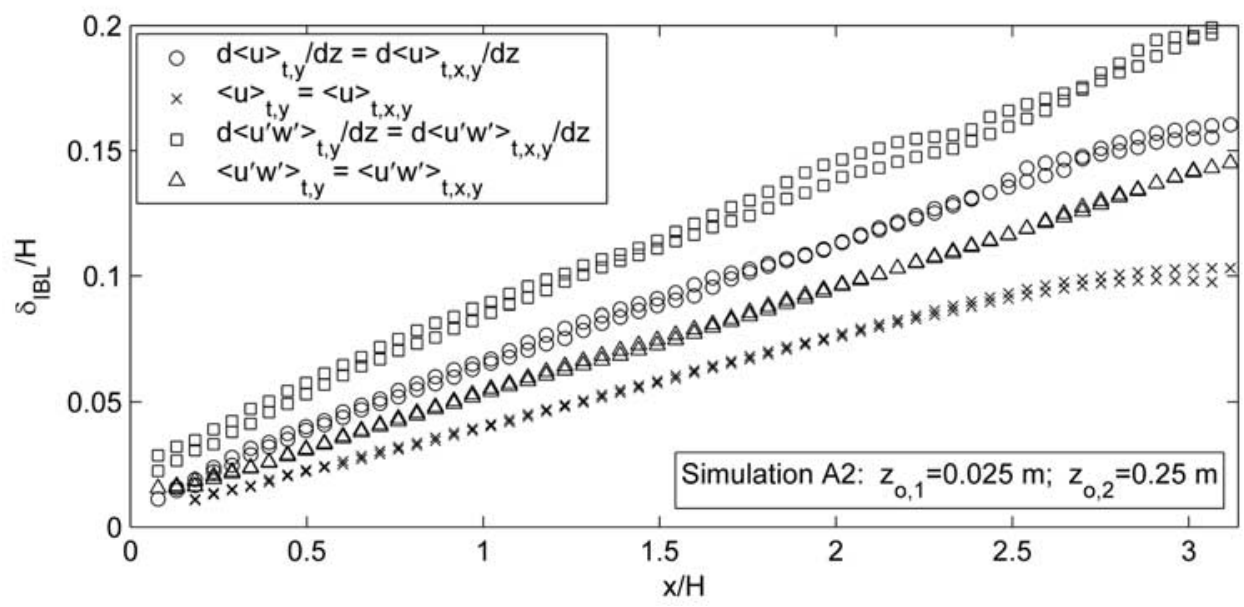

Figure A1. IBL growth for simulation A2 based on four different methods. The two lines for each method correspond to the low-to-high and high-to-low roughness transitions.

trace the internal boundary layer growth over a given patch based on the viscous diffusion analogy introduced by Miyake [1965] was positively assessed. Subsequently, a relation was developed between the blending height and the IBL thickness. Results suggest that the blending height is reached by the developing IBLs at a downstream distance equal to twice the characteristic length of the patches. IBL growth was found to be insensitive to the type of jump (high-to-low or low-to-high roughness) and to be mainly controlled by the equivalent roughness height. This allowed the formulation proposed for IBL thickness to be extended to compute blending height. The equivalent surface roughness is needed to compute the blending height using the proposed equation.

[53] Writing the total surface force over a heterogeneous area as the discrete sum of surface forces over the homogeneous patches in the area, and evaluating the resulting expression at the blending height, leads to another useful equation relating the effective surface roughness and blending height. The two equations can be combined to estimate the blending height and the equivalent surface roughness based simply on knowledge of the scales and roughness heights of the individual patches. The equivalent surface roughness computed using this model was compared to the values determined from the LES velocity profiles. Satisfactory agreement within 25\% was observed. This accuracy was significantly better than the accuracy obtained with 2 alternative formulations for the estimation of the equivalent surface roughness (Appendix B).

[54] This study provided some answers to the important questions raised in the introduction. (1) The blending of internal boundary layers formed over patches is closely related to the development of these boundary layers. (2) Surface heterogeneity has a complex effect on the landatmosphere interaction; abrupt jumps in surface characteristics induce sharp changes in the velocity profiles and discontinuities in the stress profiles close to the surface. Moreover, roughness changes invariably increase the equivalent roughness of the surface above values expected from linear accumulation of the effects of the individual patches. This is confirmed by the increase in the equivalent surface roughness for decreasing patch length, i.e., increasing number of jumps, indicating that surface heterogeneity actually amplifies the land-atmosphere interaction. (3) A succinct and realistic parameterization of blending height and effective surface roughness was developed (equations (32) and (33)) and showed good agreement with LES.

\section{Appendix A}

[55] The IBL can be determined from LES data using several methods. In this work, the $u$ velocity gradient was used. Presented below are three alternative methods.

[56] 1. IBL height defined as the point where $\langle u\rangle_{\mathrm{t}, \mathrm{y}}=$ $\langle u\rangle_{\mathrm{t}, \mathrm{x}, \mathrm{y}}$. This corresponds to the point where the velocity is equally controlled by the underlying surface and the "plume" from the patch directly upstream.

[57] 2. IBL height defined as the point where $d\left(\left\langle u^{\prime} w^{\prime}\right\rangle_{\mathrm{t}, \mathrm{y}}\right) /$ $d z=d\left(\left\langle u^{\prime} w^{\prime}\right\rangle_{\mathrm{t}, \mathrm{x}, \mathrm{y}}\right) / d z$. This corresponds to the point where the stress profile is insensitive to the underlying surface. The stress is mainly controlled by the flow from the upstream patch.

[58] 3. IBL height defined as the point where $\left\langle u^{\prime} w^{\prime}\right\rangle_{\mathrm{t}, \mathrm{y}}=$ $\left\langle u^{\prime} w^{\prime}\right\rangle_{\mathrm{t}, \mathrm{x}, \mathrm{y}}$. This corresponds to the point where the stress is equally controlled by the underlying surface and the "plume" from the patch directly upstream.

[59] Figure A1 depicts the IBL top defined using the three methods described above; results based on $d\langle u\rangle_{t, y} / d z=$ $d\langle u\rangle_{t, x, y} / d z$ are also presented for comparison.

\section{Appendix B}

[60] Several models similar to the one used here are available to compute the equivalent surface roughness using blending height. Mason [1988] proposed a model that uses equation (31) combined with

$$
h_{\mathrm{b}}\left[\ln \left(\frac{h_{\mathrm{b}}}{z_{\mathrm{o}}}\right)\right]^{2}=2 \kappa^{2} L_{\mathrm{p}} .
$$

[61] Equation (B1) is obtained from the balance between horizontal advection and vertical stress divergence (recall that the equation used in this paper is obtained from the balance between horizontal advection and vertical diffu- 


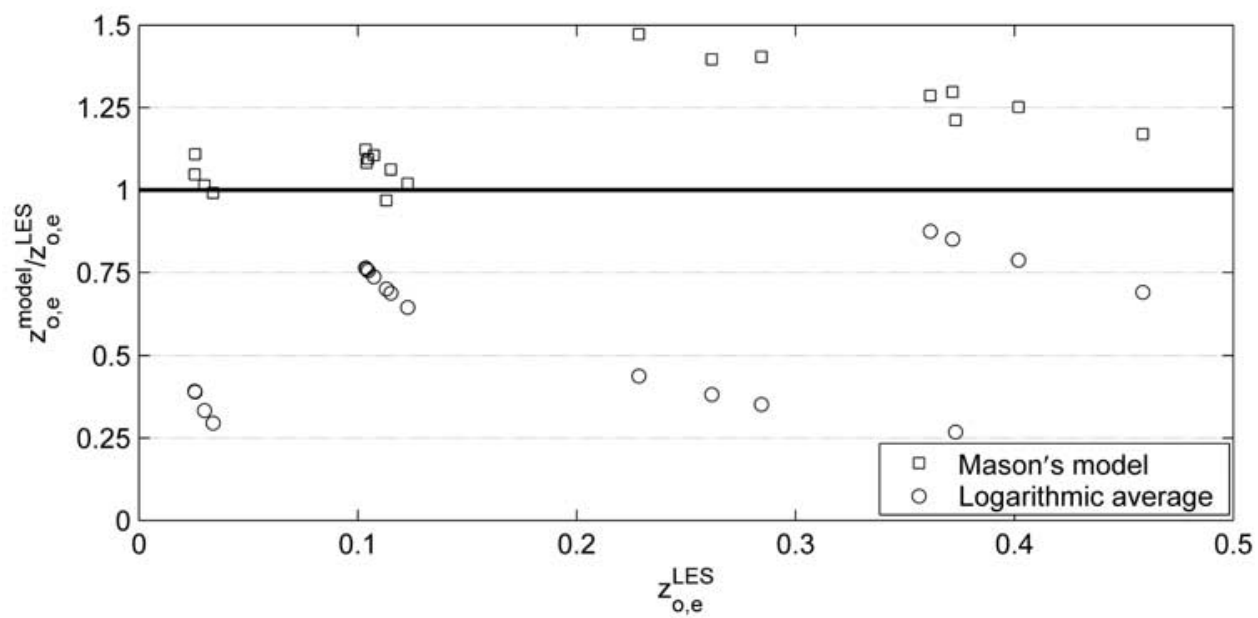

Figure B1. Comparison of equivalent surface roughness estimated by the logarithmic average model and Mason's model to LES values of roughness. The thick line corresponds to $z_{o}^{\text {model }}=z_{o}^{\text {LES }}$ (perfect fit).

sion). Comparing the predictions of that model with surface roughness values determined from LES results indicates that Mason's model regularly overpredicts $z_{\mathrm{o}, \mathrm{e}}$ by up to $50 \%$ (Figure B1). The overprediction is the highest at high $z_{\mathrm{o}, \mathrm{e}}$ and can be traced to an underprediction of the blending height by equation (B1).

[62] On the other hand, Claussen [1991] used equation (31) and estimated the height at which that equation is to be applied through an error minimization procedure. Claussen minimized the sum of the error of assuming horizontal homogeneity and the error of assuming local equilibrium of the flow with the surface. He obtained equation (B2) which is very similar to the one proposed here

$$
h_{\mathrm{b}}\left[\ln \left(\frac{h_{\mathrm{b}}}{z_{o}}\right)\right]=C_{1} \kappa L_{\mathrm{p}}
$$

[63] The equation will be different from equation (28) for short patches where the $(-1)$ term in equation (28) is significant. Claussen first proposed $C_{1}=2 \kappa=0.8$ [Claussen, 1990] but he later used RANS results and a trial and error investigation [Claussen, 1991] to conclude that the best value for $C_{1}$ is 1.75 which would yield very close results to the model in this work (here a value of 1.7 is proposed for the coefficient $C$ in equation (28)).

[64] The main alternative modeling approach (to the blending height approach discussed so far) used to estimate the equivalent surface roughness is the logarithmic average (see Taylor [1987] for a comprehensive discussion of this method) that estimates the equivalent roughness of a surface using

$$
\ln \left(z_{\mathrm{o}, \mathrm{e}}\right)=\sum_{i=1}^{N} f_{i} \ln \left(z_{\mathrm{o}, i}\right) .
$$

The equivalent surface roughness was estimated using this model and compared to LES data. Figure B1 shows comparison results. It can be seen that the error can be as large as $75 \%$ and that the log average model consistently underpredicts the effective surface roughness. These comparisons suggest that the model proposed in this paper (equations (32) and (33)) is better suited to estimate the equivalent roughness than logarithmic averaging or Mason's model, and would give results close to Claussen's model.

[65] Acknowledgments. This study was funded by NSF under EAR9909679 and by agreement R828771-0-01 from the U.S. Environmental Protection Agency's Science to Achieve Results (STAR) Program. The authors are thankful for the Scientific Computing Division of the National Center for Atmospheric Research (NCAR) for the use of their computer clusters.

\section{References}

Albertson, J. D., and M. B. Parlange (1999a), Natural integration of scalar fluxes from complex terrain, Adv. Water Resour., 23, 239-252.

Albertson, J. D., and M. B. Parlange (1999b), Surface length-scales and shear stress: Implications for land-atmosphere interaction over complex terrain, Water Resour. Res., 35(7), 2121-2132.

Andren, A., A. R. Brown, J. Graf, P. J. Mason, C. H. Moeng, F. T. M. Nieuwstadt, and U. Schumann (1994), Large-eddy simulation of a neutrally stratified boundary layer: A comparison of four computer codes, Q. J. R. Meteorol. Soc., 120, 1457-1484.

Antonia, R. A., and R. E. Luxton (1971), The response of a turbulent boundary layer to a step change in surface roughness. part 1. Smooth to rough, J. Fluid Mech., 48, 721-761.

Antonia, R. A., and R. E. Luxton (1972), The response of a turbulent boundary layer to a step change in surface roughness. part 2. Rough to smooth, J. Fluid Mech., 53, 735-757.

Avissar, R. (1991), A statistical-dynamical approach to parameterize subgrid-scale land-surface heterogeneity in climate models, Surv. Geophys., $12,155-178$.

Avissar, R. (1992), Conceptual aspects of a statistical-dynamical approach to represent landscape subgrid-scale heterogeneities in atmospheric models, J. Geophys. Res., 97, 2729-2742.

Avissar, R., and R. A. Pielke (1989), A parameterization of heterogeneous land surfaces for atmospheric numerical models and its impact on regional meteorology, Mon. Weather Rev., 117, 2113-2136.

Avissar, R., E. W. Eloranta, K. Gurer, and G. J. Tripoli (1998), An evaluation of the large-eddy simulation option of the regional modeling system in simulating a convective boundary layer: A FIFE case study, J. Atmos. Sci., 55, 1109-1130.

Bradley, E. F. (1968), A micrometeorological study of velocity profiles and surface drag in the region modified by a change in surface roughness, Q. J. R. Meteorol. Soc., 94, 361-379.

Brutsaert, W. (1998), Land-surface water vapor and sensible heat flux: Spatial variability, homogeneity, and measurement scales, Water Resour. Res., 34(10), 2433-2442.

Brutsaert, W., and M. B. Parlange (1992), The unstable surface layer above forest: Regional evaporation and heat flux, Water Resour. Res., 28(12), $3129-3134$ 
Brutsaert, E., M. B. Parlange, and J. H. C. Gash (1989), Neutral humidity profiles in boundary layer and regional evaporation from sparse pine forest, Ann. Geophys., 7, 623-630.

Claussen, M. (1990), Area-averaging of surface fluxes in a neutrally stratified, horizontally inhomogeneous atmospheric boundary layer, Atmos. Environ. Part A, 24(6), 1349-1360.

Claussen, M. (1991), Estimation of areally-averaged surface fluxes, Boundary Layer Meteorol., 54, 387-410.

Garratt, J. R. (1990), The internal boundary layer-A review, Boundary Layer Meteorol., 50, 171-203.

Germano, M., U. Piomelli, P. Moin, and W. Cabot (1991), A dynamic subgrid-scale eddy viscosity model, Phys. Fluids A, 3, 1760-1765.

Ghosal, S., T. S. Lund, P. Moin, and K. Akselvoll (1995), A dynamic localization model for large eddy simulation of turbulent flows, J. Fluid Mech., 286, 229-255.

Glendening, J. W., and C. L. Lin (2002), Large eddy simulation of internal boundary layers created by a change in surface roughness, J. Atmos. Sci., 59, 1697-1711.

Goode, K., and E. Belcher (1999), On the parameterization of the effective roughness length for momentum transfer over heterogeneous terrain, Boundary Layer Meteorol., 93, 133-154.

Hechtel, L. M., R. B. Stull, and C.-H. Moeng (1990), The effects of nonhomogeneous surface fluxes on the convective boundary-layer: A case study using large-eddy simulation, J. Atmos. Sci., 47, 1721 1741.

Intergovernmental Panel on Climate Change (1997), An Introduction to Simple Climate Models Used in the IPCC Second Assessment Report, Technical Paper, Working Group I, edited by J. T. Houghton et al., Cambridge Univ. Press, New York.

Katul, G. G., C. R. Chu, M. B. Parlange, J. D. Albertson, and T. A. Ortenburger (1995), The low-wavenumber spectral characteristics of velocity and temperature in the atmospheric surface layer, J. Geophys. Res., 100, 14,243-14,255.

Khanna, S., and J. B. Brasseur (1997), Analysis of Monin-Obukhov similarity from large-eddy simulation, J. Fluid Mech., 345, 251-286.

Koster, R. D., and M. J. Suarez (1992), Modeling the land surface boundary in climate models as a composite of independent vegetation stands, J. Geophys. Res., 97, 2697-2715.

Lilly, D. K. (1967), The representation of small scale turbulence in numerical simulation experiments, in Proceedings of IBM Scientific Computing Symposium on Environmental Sciences, p. 195, IBM Data Process. Div. Whiteplains, N. Y.

Mason, P. J. (1988), The formation of areally-averaged roughness lengths, Q. J. R. Meteorol. Soc., 114, 399-420.

Mason, P. J., and N. S. Callen (1986), On the magnitude of the subgridscale eddy coefficient in large-eddy simulations of turbulent channel flow, J. Fluid Mech., 162, 439-462.

Mason, P. J., and D. J. Thomson (1992), Stochastic backscatter in largeeddy simulations of boundary layers, J. Fluid Mech., 242, 51-78.

Meneveau, C., and J. Katz (2000), Scale-invariance and turbulence models for large-eddy simulation, Annu. Rev. Fluid Mech., 32, 1-32.

Meneveau, C., T. Lund, and W. Cabot (1996), A Lagrangian dynamic subgrid-scale model of turbulence, J. Fluid Mech., 319, 353-385.

Miyake, M. (1965), Transformation of the atmospheric boundary layer over inhomogeneous surfaces, Rep. AD 626630, Dep. Atmos. Sci., Univ. of Wash., Seattle.

Moeng, C. H. (1984), A large-eddy simulation model for the study of planetary boundary-layer turbulence, J. Atmos. Sci., 46, 2311-2330.
Monin, A. S., and A. M. Obukhov (1954), Basic laws of turbulent mixing in the ground layer of the atmosphere (in Russian), Tr. Geofiz. Inst. Akad. Nauk SSSR, 151, 163-187.

Orszag, S. S. (1970), Transform method for calculation of vector coupled sums: Application to the spectral form of the vorticity equation, J. Atmos. Sci., 27, 890-895.

Orszag, S. A., and Y. H. Pao (1974), Numerical computation of turbulent shear flows, Adv. Geophys., 18, 224-236.

Parlange, M. B., and W. Brutsaert (1989), Regional roughness of the land forest and surface shear stress under neutral conditions, Boundary Layer Meteorol., 48, 69-81.

Parlange, M. B., and W. Brutsaert (1993), Regional shear stress of broken forest from radiosonde wind profiles in the unstable surface layer, Boundary Layer Meteorol., 64, 355-368.

Parlange, M. B., W. E. Eichinger, and J. D. Albertson (1995), Regional evaporation into the atmospheric boundary layer, Rev. Geophys., 33(1), 99-124.

Perry, A. E., K. L. Lim, and S. M. Henbest (1987), An experimental study of the turbulence structure in smooth- and rough-wall boundary layers, J. Fluid Mech., 177, 437-466.

Porté-Agel, F., C. Meneveau, and M. B. Parlange (2000), A scale-dependent dynamic model for large-eddy simulation: Application to a neutral atmospheric boundary layer, J. Fluid Mech., 415, 261-284.

Rao, K. S., J. C. Wyngaard, and O. R. Coté (1973), The structure of the two-dimensional internal boundary layer over a sudden change of surface roughness, J. Atmos. Sci., 31, 738-746.

Schmid, H. P., and B. Bünzly (1995), The influence of surface texture on the effective roughness length, Q. J. R. Meteorol. Soc., 121, 1-21.

Schmidt, H., and U. Schumann (1989), Coherent structures of the convective boundary layer derived from large-eddy simulations, J. Fluid Mech., 200, 511-562.

Shen, S. H., and M. Y. Leclerc (1995), How large must surface inhomogeneities be before they influence the convective boundary layer structures? A case study, O. J. R. Meteorol. Soc., 121, 1209-1228.

Smagorinsky, J. S. (1963), General circulation experiments with the primitive equations: part I. The basic experiment, Mon. Weather Rev., 91, 99-164.

Taylor, P. A. (1987), Comments and further analysis on effective roughness lengths for use in numerical three-dimensional models, Boundary Layer Meteorol., 39, 403-418.

Wieringa, J. (1976), An objective exposure correction method for average wind speeds measured at a shelter location, Q. J. R. Meteorol. Soc., 102, $241-253$.

Wieringa, J. (1986), Roughness-dependent geographical interpolation of surface wind speed averages, Q. J. R. Meteorol. Soc., 112, 867-889.

Wood, N., and P. Mason (1991), The influence of the static stability on the effective roughness lengths for momentum and heat transfer, $Q . J . R$. Meteorol. Soc., 117, 1025-1056.

E. Bou-Zeid and M. B. Parlange, Department of Geography and Environmental Engineering and Center for Environmental and Applied Fluid Mechanics, Johns Hopkins University, 3400 North Charles street, 313 Ames Hall, Baltimore, MD 21218, USA. (eliebz@jhu.edu; mbparlange@ jhu.edu)

C. Meneveau, Department of Mechanical Engineering and Center for Environmental and Applied Fluid Mechanics, Johns Hopkins University, 3400 North Charles Street, 223 Latrobe Hall, Baltimore, MD 21218, USA. (meneveau@jhu.edu) 